\title{
Article \\ Some Generalizations of Different Types of Quantum Integral Inequalities for Differentiable Convex Functions with Applications
}

\author{
Dafang Zhao ${ }^{1,2}{ }^{\mathbb{D}}$, Muhammad Aamir Ali ${ }^{3}\left(\mathbb{D}\right.$, Waewta Luangboon ${ }^{4}$, Hüseyin Budak ${ }^{5}$ \\ and Kamsing Nonlaopon $4, *$ (D)
}

1 School of Mathematics and Statistics, Hubei Normal University, Huangshi 435002, China; dafangzhao@163.com

2 Northwest Institute of Eco-Environment and Resources, Chinese Academy of Sciences, Lanzhou 730000, China

3 Jiangsu Key Laboratory for NSLSCS, School of Mathematical Sciences, Nanjing Normal University, Nanjing 210023, China; mahr.muhammad.aamir@gmail.com

4 Department of Mathematics, Faculty of Science and Arts, Khon Kaen University, Khon Kaen 40002, Thailand; waewta_l@kkumail.com

5 Department of Mathematics, Faculty of Science and Arts, Düzce University, Düzce 81620, Turkey; hsyn.budak@gmail.com

* Correspondence: nkamsi@kku.ac.th

check for updates

Citation: Zhao, D.; Ali, M.A.;

Luangboon, W.; Budak, H.;

Nonlaopon, K. Some Generalizations of Different Types of Quantum Integral Inequalities for Differentiable Convex Functions with Applications. Fractal Fract. 2022, 6,

129. https://doi.org/10.3390/

fractalfract6030129

Academic Editor: Ahmed I. Zayed

Received: 6 January 2022

Accepted: 18 February 2022

Published: 23 February 2022

Publisher's Note: MDPI stays neutral with regard to jurisdictional claims in published maps and institutional affiliations.

Copyright: (C) 2022 by the authors. Licensee MDPI, Basel, Switzerland. This article is an open access article distributed under the terms and conditions of the Creative Commons Attribution (CC BY) license (https:// creativecommons.org/licenses/by/ $4.0 /)$.

\begin{abstract}
In this paper, we prove a new quantum integral equality involving a parameter, left and right quantum derivatives. Then, we use the newly established equality and prove some new estimates of quantum Ostrowski, quantum midpoint, quantum trapezoidal and quantum Simpson's type inequalities for $q$-differentiable convex functions. It is also shown that the newly established inequalities are the refinements of the existing inequalities inside the literature. Finally, some examples and applications are given to illustrate the investigated results.
\end{abstract}

Keywords: midpoint inequalities; trapezoidal inequalities; Ostrowski's inequalities; Simpson's inequalities; quantum calculus; convex functions

\section{Introduction}

A. Ostrowski showed an inequality involving a function with bounded derivative in 1938, which became known as the Ostrowski inequality [1]. The following is the inequality:

Theorem 1. For a differentiable functions $\mathfrak{F}:\left[\mathfrak{y}_{1}, \mathfrak{y}_{2}\right] \rightarrow \mathbb{R}$ over $\left(\mathfrak{y}_{1}, \mathfrak{y}_{2}\right)$ with $|\mathfrak{F}(\mathfrak{r})| \leq M$, the following inequality holds:

$$
\left|\mathfrak{F}(\mathfrak{r})-\frac{1}{\mathfrak{y}_{2}-\mathfrak{y}_{1}} \int_{\mathfrak{y}_{1}}^{\mathfrak{y}_{2}} \mathfrak{F}(t) d t\right| \leq M\left(\mathfrak{y}_{2}-\mathfrak{y}_{1}\right)\left[\frac{\left(\mathfrak{r}-\mathfrak{y}_{1}\right)^{2}+\left(\mathfrak{y}_{2}-\mathfrak{r}\right)^{2}}{2}\right],
$$

where $\mathfrak{r} \in\left[\mathfrak{y}_{1}, \mathfrak{y}_{2}\right]$.

The following are two possible interpretations of the Ostrowski inequality:

(i) Estimation of the functional value's deviation from its average value.

(ii) A rectangle is used to approximate the area under the curve.

On the other hand, Budak et al. proved the quantum version of the inequality (1) as follows: 
Theorem 2 ([2]). Let $\mathfrak{F}:\left[\mathfrak{y}_{1}, \mathfrak{y}_{2}\right] \rightarrow \mathbb{R}$ be a q-differentiable function. If $\mathfrak{y}_{1} D_{q} \mathfrak{F}$ and $\mathfrak{y}_{2} D_{q} \mathfrak{F}$ are continuous and integrable on $\left[\mathfrak{y}_{1}, \mathfrak{y}_{2}\right]$ with $\left|\mathfrak{y}_{1} D_{q} \mathfrak{F}\right|,\left|\mathfrak{y}_{2} D_{q} \mathfrak{F}\right| \leq M$, then the following inequality holds for $\mathfrak{r} \in\left[\mathfrak{y}_{1}, \mathfrak{y}_{2}\right]$ :

$$
\begin{aligned}
& \left|\mathfrak{F}(\mathfrak{r})-\frac{1}{\mathfrak{y}_{2}-\mathfrak{y}_{1}}\left[\int_{\mathfrak{y}_{1}}^{\mathfrak{r}} \mathfrak{F}(t) \mathfrak{y}_{1} d_{q} t+\int_{\mathfrak{r}}^{\mathfrak{y}_{2}} \mathfrak{F}(t) \mathfrak{y}_{2} d_{q} t\right]\right| \\
& \leq \frac{q M}{\left(\mathfrak{y}_{2}-\mathfrak{y}_{1}\right)}\left[\frac{\left(\mathfrak{r}-\mathfrak{y}_{1}\right)^{2}+\left(\mathfrak{y}_{2}-\mathfrak{r}\right)^{2}}{[2]_{q}}\right] .
\end{aligned}
$$

It is also well known that $\mathfrak{F}$ is convex if and only if it satisfies the Hermite-Hadamard inequality, stated below:

$$
\mathfrak{F}\left(\frac{\mathfrak{y}_{1}+\mathfrak{y}_{2}}{2}\right) \leq \frac{1}{\mathfrak{y}_{2}-\mathfrak{y}_{1}} \int_{\mathfrak{y}_{1}}^{\mathfrak{y}_{2}} \mathfrak{F}(\mathfrak{r}) d \mathfrak{r} \leq \frac{\mathfrak{F}\left(\mathfrak{y}_{1}\right)+\mathfrak{F}\left(\mathfrak{y}_{2}\right)}{2}
$$

where $\mathfrak{F}: \mathfrak{I} \rightarrow \mathbb{R}$ is a convex function and $\mathfrak{y}_{1}, \mathfrak{y}_{2} \in \mathfrak{I}$ with $\mathfrak{y}_{1}<\mathfrak{y}_{2}$. For some recent developments of inequality (3), one can consult [3-6].

In [7], Alp et al. proved the following version of quantum Hermite-Hadamard type for convex functions using the left quantum integrals:

Theorem 3. For any convex function $\mathfrak{F}:\left[\mathfrak{y}_{1}, \mathfrak{y}_{2}\right] \rightarrow \mathbb{R}$, the following inequality holds:

$$
\mathfrak{F}\left(\frac{q \mathfrak{y}_{1}+\mathfrak{y}_{2}}{[2]_{q}}\right) \leq \frac{1}{\mathfrak{y}_{2}-\mathfrak{y}_{1}} \int_{\mathfrak{y}_{1}}^{\mathfrak{y}_{2}} \mathfrak{F}(\mathfrak{r})_{\mathfrak{y}_{1}} d_{q} \mathfrak{r} \leq \frac{q \mathfrak{F}\left(\mathfrak{y}_{1}\right)+\mathfrak{F}\left(\mathfrak{y}_{2}\right)}{[2]_{q}}
$$

Recently, Bermudo et al. [8] used the right quantum integrals and proved the following variant of Hermite-Hadamard type inequalities for convex functions:

Theorem 4. For any convex function $\mathfrak{F}:\left[\mathfrak{y}_{1}, \mathfrak{y}_{2}\right] \rightarrow \mathbb{R}$, the following inequalities holds:

$$
\begin{gathered}
\mathfrak{F}\left(\frac{\mathfrak{y}_{1}+q \mathfrak{y}_{2}}{[2]_{q}}\right) \leq \frac{1}{\mathfrak{y}_{2}-\mathfrak{y}_{1}} \int_{\mathfrak{y}_{1}}^{\mathfrak{y}_{2}} \mathfrak{F}(\mathfrak{r})^{\mathfrak{y}_{2}} d_{q} \mathfrak{r} \leq \frac{\mathfrak{F}\left(\mathfrak{y}_{1}\right)+q \mathfrak{F}\left(\mathfrak{y}_{2}\right)}{[2]_{q}} \\
\mathfrak{F}\left(\frac{\mathfrak{y}_{1}+\mathfrak{y}_{2}}{2}\right) \leq \frac{1}{2\left(\mathfrak{y}_{2}-\mathfrak{y}_{1}\right)}\left[\int_{\mathfrak{y}_{1}}^{\mathfrak{y}_{2}} \mathfrak{F}(\mathfrak{r})_{\mathfrak{y}_{1}} d_{q} \mathfrak{r}+\int_{\mathfrak{y}_{1}}^{\mathfrak{y}_{2}} \mathfrak{F}(\mathfrak{r})^{\mathfrak{y}_{2}} d_{q} \mathfrak{r}\right] \leq \frac{\mathfrak{F}\left(\mathfrak{y}_{1}\right)+\mathfrak{F}\left(\mathfrak{y}_{2}\right)}{2} .
\end{gathered}
$$

Remark 1. It is obvious that if we take the limit as $q \rightarrow 1^{-}$in (4)-(6), then we obtain the inequality (3).

In the literature there are several papers focused on obtaining some bounds for the left and right estimates of inequalities (4) and (5). For example, Noor et al. [9] and Alp et al. [7] gave some bounds for the right side and right side of the inequality (4), respectively, whereas Budak established similar bounds for the left and right side of the inequality (5) in [10]. In [11], Liu and Zhuang obtained some trapezoid type inequalities by using the twice $q$-differentiable functions. The authors proved several new estimates by utilizing the generalized convex functions in [12-14]. Brahim et al. established some new version of quantum Hermite-Hadamard inequality in [15]. On the other hand, some papers were devoted to fractional post-quantum inequalities [16,17]. Some authors generalized the quantum Hermite-Hadamard inequalities for coordinated convex functions in [18-20]. In [21-23], the authors used convexity and coordinated convexity to prove some Simpson's and Newton's type inequalities via $q$-calculus. For the study of Ostrowski's inequalities, one can consult $[24,25]$. The quantum version of Bernoulli inequality is given by Alomari in [26]. 
Inspired by the ongoing studies, we prove a new parameterized quantum integral identity involving left and right quantum derivatives to prove different variants of quantum integral inequalities for quantum differentiable convex functions. The main advantage of the newly established inequalities is that these can be turned into quantum Ostrowski's type inequalities for convex functions [2], classical Ostrowski's type inequalities for convex functions [27], several classical integral inequalities for convex functions [28] and several new quantum integral inequalities such as midpoint type, trapezoidal type, Ostrowski's type and Simpson's type without having to prove each one separately.

The following is the structure of this paper: Section 2 provides a brief overview of the fundamentals of $q$-calculus as well as other related studies in this field. In Section 3, we establish an identity that plays an essential role in developing the main results of this paper. The different variants of quantum integral inequalities for quantum differentiable convex functions are described in Section 4. The relationship between the findings reported here and similar findings in the literature are also taken into account. In Section 5, we show some examples to illustrate the investigated results. In Section 6, we provide some applications to special means of real numbers by using the newly established results. Section 7 concludes with some recommendations for future research.

\section{Preliminaries of $q$-Calculus and Some Inequalities}

In this section, we first present the definitions and some properties of quantum derivatives and quantum integrals. We also mention some well-known inequalities for quantum integrals. Throughout this paper, let $0<q<1$ be a constant.

The $q$-number or $q$-analog of $n \in \mathbb{N}$ is given by

$$
[n]_{q}=\frac{1-q^{n}}{1-q}=1+q+q^{2}+\cdots+q^{n-1} .
$$

Definition 1 ([29]). Let $\mathfrak{F}:\left[\mathfrak{y}_{1}, \mathfrak{y}_{2}\right] \rightarrow \mathbb{R}$ be a continuous function. Then, the left $q$-derivative of function $\mathfrak{F}$ at $\mathfrak{r} \in\left[\mathfrak{y}_{1}, \mathfrak{y}_{2}\right]$ is defined by

$$
\mathfrak{y}_{1} D_{q} \mathfrak{F}(\mathfrak{r})= \begin{cases}\frac{\mathfrak{F}(\mathfrak{r})-\mathfrak{F}\left(q \mathfrak{r}+(1-q) \mathfrak{y}_{1}\right)}{(1-q)\left(\mathfrak{r}-\mathfrak{y}_{1}\right)}, & \text { if } \mathfrak{r} \neq \mathfrak{y}_{1} \\ \lim _{\mathfrak{r} \rightarrow \mathfrak{y}_{1}} \mathfrak{y}_{1} D_{q} \mathfrak{F}(\mathfrak{r}), & \text { if } \mathfrak{r}=\mathfrak{y}_{1} .\end{cases}
$$

The function $\mathfrak{F}$ is said to be $q$-differentiable function on $\left[\mathfrak{y}_{1}, \mathfrak{y}_{2}\right]$ if $\mathfrak{y}_{1} D_{q} \mathfrak{F}(\mathfrak{r})$ exists for all $\mathfrak{r} \in\left[\mathfrak{y}_{1}, \mathfrak{y}_{2}\right]$.

Note that, if $\mathfrak{y}_{1}=0$ and ${ }_{0} D_{q} \mathfrak{F}(\mathfrak{r})=D_{q} \mathfrak{F}(\mathfrak{r})$, then (8) reduces to

$$
D_{q} \mathfrak{F}(\mathfrak{r})= \begin{cases}\frac{\mathfrak{F}(\mathfrak{r})-\mathfrak{F}(q \mathfrak{r})}{(1-q) \mathfrak{r}}, & \text { if } \mathfrak{r} \neq 0 ; \\ \lim _{\mathfrak{r} \rightarrow 0} D_{q} \mathfrak{F}(\mathfrak{r}), & \text { if } \mathfrak{r}=0,\end{cases}
$$

which is the $q$-Jackson derivative, see [29-31] for more details.

Theorem 5 ([29]). If $\mathfrak{F}, g: J \rightarrow \mathbb{R}$ are q-differentiable functions, then the following identities hold:

(i) The product $\mathfrak{F g}:\left[\mathfrak{y}_{1}, \mathfrak{y}_{2}\right] \rightarrow \mathbb{R}$ is q-differentiable on $\left[\mathfrak{y}_{1}, \mathfrak{y}_{2}\right]$ with

$$
\begin{aligned}
\mathfrak{y}_{1} D_{q}(\mathfrak{F} g)(\mathfrak{r}) & =\mathfrak{F}(\mathfrak{r})_{\mathfrak{y}_{1}} D_{q} g(\mathfrak{r})+g(q \mathfrak{r}+(1-q) \mathfrak{r})_{\mathfrak{y}_{1}} D_{q} \mathfrak{F}(\mathfrak{r}) \\
& =g(\mathfrak{r})_{\mathfrak{y}_{1}} D_{q} \mathfrak{F}(\mathfrak{r})+\mathfrak{F}(q \mathfrak{r}+(1-q) \mathfrak{r})_{\mathfrak{y}_{1}} D_{q} g(\mathfrak{r})
\end{aligned}
$$

(ii) If $g(\mathfrak{r}) g(q \mathfrak{r}+(1-q) \mathfrak{r}) \neq 0$, then $\mathfrak{F} / g$ is $q$-differentiable on $\left[\mathfrak{y}_{1}, \mathfrak{y}_{2}\right]$ with

$$
\mathfrak{y}_{1} D_{q}\left(\frac{\mathfrak{F}}{g}\right)(\mathfrak{r})=\frac{g(\mathfrak{r})_{\mathfrak{y}} D_{q} \mathfrak{F}(\mathfrak{r})-\mathfrak{F}(\mathfrak{r})_{\mathfrak{y}_{1}} D_{q} g(\mathfrak{r})}{g(\mathfrak{r}) g(q \mathfrak{r}+(1-q) \mathfrak{r})} .
$$


Definition 2 ([29]). Let $\mathfrak{F}:\left[\mathfrak{y}_{1}, \mathfrak{y}_{2}\right] \rightarrow \mathbb{R}$ be a continuous function. Then, the left $q$-integral of function $\mathfrak{F}$ at $z \in\left[\mathfrak{y}_{1}, \mathfrak{y}_{2}\right]$ is defined by

$$
\int_{\mathfrak{y}_{1}}^{z} \mathfrak{F}(\mathfrak{r})_{\mathfrak{y}_{1}} d_{q} \mathfrak{r}=(1-q)\left(z-\mathfrak{y}_{1}\right) \sum_{n=0}^{\infty} q^{n} \mathfrak{F}\left(q^{n} z+\left(1-q^{n}\right) \mathfrak{y}_{1}\right) .
$$

The function $\mathfrak{F}$ is said to be q-integrable function on $\left[\mathfrak{y}_{1}, \mathfrak{y}_{2}\right]$ if $\int_{\mathfrak{y}_{1}}^{z} \mathfrak{F}(\mathfrak{r})_{\mathfrak{y}_{1}} d_{q} \mathfrak{r}$ exists for all $z \in\left[\mathfrak{y}_{1}, \mathfrak{y}_{2}\right]$.

Note that, if $\mathfrak{y}_{1}=0$, then (9) reduces to

$$
\int_{0}^{z} \mathfrak{F}(\mathfrak{r})_{0} d_{q} \mathfrak{r}=\int_{0}^{z} \mathfrak{F}(\mathfrak{r}) d_{q} \mathfrak{r}=(1-q) z \sum_{n=0}^{\infty} q^{n} \mathfrak{F}\left(q^{n} z\right),
$$

which is the $q$-Jackson integral, see [29-31] for more details.

Theorem 6 ([29]). If $\mathfrak{F}:\left[\mathfrak{y}_{1}, \mathfrak{y}_{2}\right] \rightarrow \mathbb{R}$ is a continuous function and $z \in\left[\mathfrak{y}_{1}, \mathfrak{y}_{2}\right]$, then the following identities hold:

(i) $\quad \mathfrak{y}_{1} D_{q} \int_{\mathfrak{y}_{1}}^{z} \mathfrak{F}(\mathfrak{r})_{\mathfrak{y}_{1}} d_{\mathfrak{q}} \mathfrak{r}=\mathfrak{F}(z)$;

(ii) $\int_{\mathcal{c}}^{z} \mathfrak{y}_{1} D_{q} \mathfrak{F}(\mathfrak{r})_{\mathfrak{y}_{1}} d_{q} \mathfrak{r}=\mathfrak{F}(z)-\mathfrak{F}(c)$ for $c \in\left(\mathfrak{y}_{1}, z\right)$.

On the other hand, Bermudo et al. [8] defined a new quantum derivative and quantum integral, which are called right $q$-derivative and right $q$-integral as follows:

Definition 3 ([8]). The right q-derivative of mapping $\mathfrak{F}:\left[\mathfrak{y}_{1}, \mathfrak{y}_{2}\right] \rightarrow \mathbb{R}$ is defined as:

$$
{ }^{\mathfrak{y}_{2}} D_{q} \mathfrak{F}(\mathfrak{r})=\frac{\mathfrak{F}\left(q \mathfrak{r}+(1-q) \mathfrak{y}_{2}\right)-\mathfrak{F}(\mathfrak{r})}{(1-q)\left(\mathfrak{y}_{2}-\mathfrak{r}\right)}, \quad \mathfrak{r} \neq \mathfrak{y}_{2} .
$$

If $\mathfrak{r}=\mathfrak{y}_{2}$, we define ${ }^{\mathfrak{y}_{2}} D_{q} \mathfrak{F}\left(\mathfrak{y}_{2}\right)=\lim _{\mathfrak{r} \rightarrow \mathfrak{y}_{2}}{ }^{\mathfrak{y}_{2}} D_{q} \mathfrak{F}(\mathfrak{r})$ if it exists and it is finite.

Definition 4 ([8]). The right $q$-definite integral of mapping $\mathfrak{F}:\left[\mathfrak{y}_{1}, \mathfrak{y}_{2}\right] \rightarrow \mathbb{R}$ on $\left[\mathfrak{y}_{1}, \mathfrak{y}_{2}\right]$ is defined as:

$$
\int_{\mathfrak{y}_{1}}^{\mathfrak{y}_{2}} \mathfrak{F}(\mathfrak{r})^{\mathfrak{y}_{2}} d_{q} \mathfrak{r}=(1-q)\left(\mathfrak{y}_{2}-\mathfrak{y}_{1}\right) \sum_{k=0}^{\infty} q^{k} \mathfrak{F}\left(q^{k} \mathfrak{y}_{1}+\left(1-q^{k}\right) \mathfrak{y}_{2}\right) .
$$

Lemma 1 ([32]). For continuous functions $\mathfrak{F}, g:\left[\mathfrak{y}_{1}, \mathfrak{y}_{2}\right] \rightarrow \mathbb{R}$, the following equality holds for $c \in\left[\mathfrak{y}_{1}, \mathfrak{y}_{2}\right]:$

$$
\begin{aligned}
& \int_{0}^{c} g(t)_{\mathfrak{y}_{1}} D_{q} \mathfrak{F}\left(t \mathfrak{y}_{2}+(1-t) \mathfrak{y}_{1}\right) d_{q} t \\
= & \left.\frac{g(t) \mathfrak{F}\left(t \mathfrak{y}_{2}+(1-t) \mathfrak{y}_{1}\right)}{\mathfrak{y}_{2}-\mathfrak{y}_{1}}\right|_{0} ^{c}-\frac{1}{\mathfrak{y}_{2}-\mathfrak{y}_{1}} \int_{0}^{c} D_{q} g(t) \mathfrak{F}\left(q t \mathfrak{y}_{2}+(1-q t) \mathfrak{y}_{1}\right) d_{q} t .
\end{aligned}
$$


Proof. From the fundamental rules of $q$-integrals and derivatives, we have

$$
\begin{aligned}
& \int_{0}^{c} g(t) \mathfrak{y}_{1} D_{q} \mathfrak{F}\left(t \mathfrak{y}_{2}+(1-t) \mathfrak{y}_{1}\right) d_{q} t \\
= & \int_{0}^{c} g(t) \frac{\mathfrak{F}\left(t \mathfrak{y}_{2}+(1-t) \mathfrak{y}_{1}\right)-\mathfrak{F}\left(q t \mathfrak{y}_{2}+(1-q t) \mathfrak{y}_{1}\right)}{(1-q)\left(\mathfrak{y}_{2}-\mathfrak{y}_{1}\right) t} d_{q} t \\
= & \frac{1}{\mathfrak{y}_{2}-\mathfrak{y}_{1}}\left[\sum_{k=0}^{\infty} g\left(q^{k} c\right) \mathfrak{F}\left(q^{k} c \mathfrak{y}_{2}+\left(1-q^{k} c\right) \mathfrak{y}_{1}\right)-\sum_{k=0}^{\infty} g\left(q^{k} c\right) \mathfrak{F}\left(q^{k+1} c \mathfrak{y}_{2}+\left(1-q^{k+1} c\right) \mathfrak{y}_{1}\right)\right] \\
= & \frac{1}{\mathfrak{y}_{2}-\mathfrak{y}_{1}} \sum_{k=0}^{\infty}\left[g\left(q^{k} c\right) \mathfrak{F}\left(q^{k} c \mathfrak{y}_{2}+\left(1-q^{k} c\right) \mathfrak{y}_{1}\right)-g\left(q^{k} c\right) \mathfrak{F}\left(q^{k+1} c \mathfrak{y}_{2}+\left(1-q^{k+1} c\right) \mathfrak{y}_{1}\right)\right] \\
= & -\frac{1}{\mathfrak{y}_{2}-\mathfrak{y}_{1}} \sum_{k=0}^{\infty}\left(g\left(q^{k} c\right)-g\left(q^{k+1} c\right)\right) \mathfrak{F}\left(q^{k+1} c \mathfrak{y}_{2}+\left(1-q^{k+1} c\right) \mathfrak{y}_{1}\right) \\
= & \frac{1}{\mathfrak{y}_{2}-\mathfrak{y}_{1}}\left[g(c) \mathfrak{F}\left(c \mathfrak{y}_{2}+(1-c) \mathfrak{y}_{1}\right)-g(0) \mathfrak{F}\left(\mathfrak{y}_{1}\right)\right] \\
= & \left.\frac{c}{\mathfrak{y}_{2}-\mathfrak{y}_{1}} q^{k} D_{q} g\left(q^{k} c\right) \mathfrak{F}\left(q^{k+1} c \mathfrak{y}_{2}+(1-t) q^{k+1} c\right) \mathfrak{y}_{1}\right) \\
\mathfrak{y}_{2}-\mathfrak{y}_{1} & \left.\right|_{0} ^{c}-\frac{1}{\mathfrak{y}_{2}-\mathfrak{y}_{1}} \int_{0}^{c} D_{q} g(t) \mathfrak{F}\left(q t \mathfrak{y}_{2}(1-q t) \mathfrak{y}_{2}\right) d_{q} t .
\end{aligned}
$$

Lemma 2 ([33]). For continuous functions $\mathfrak{F}, g:\left[\mathfrak{y}_{1}, \mathfrak{y}_{2}\right] \rightarrow \mathbb{R}$, the following equality holds for $c \in\left[\mathfrak{y}_{1}, \mathfrak{y}_{2}\right]:$

$$
\begin{aligned}
& \int_{0}^{c} g(t)^{\mathfrak{y}_{2}} D_{q} \mathfrak{F}\left(t \mathfrak{y}_{1}+(1-t) \mathfrak{y}_{2}\right) d_{q} t \\
= & \frac{1}{\mathfrak{y}_{2}-\mathfrak{y}_{1}} \int_{0}^{c} D_{q} g(t) \mathfrak{F}\left(q t \mathfrak{y}_{1}+(1-q t) \mathfrak{y}_{2}\right) d_{q} t-\left.\frac{g(t) \mathfrak{F}\left(t \mathfrak{y}_{1}+(1-t) \mathfrak{y}_{2}\right)}{\mathfrak{y}_{2}-\mathfrak{y}_{1}}\right|_{0} ^{c} .
\end{aligned}
$$

\section{Identities}

In this section, we establish a general version of quantum integral equality using the integration by parts method for quantum integrals to obtain the main results.

Lemma 3. Let $\mathfrak{F}:\left[\mathfrak{y}_{1}, \mathfrak{y}_{2}\right] \rightarrow \mathbb{R}$ be a $q$-differentiable function. If $\mathfrak{y}_{1} D_{q} \mathfrak{F}$ and $\mathfrak{y}_{2} D_{q} \mathfrak{F}$ are continuous and integrable on $\left[\mathfrak{y}_{1}, \mathfrak{y}_{2}\right]$, then for $\mathfrak{r} \in\left[\mathfrak{y}_{1}, \mathfrak{y}_{2}\right]$ and $\lambda \geq 0$ one has the identity:

$$
\begin{aligned}
& (1-\lambda) \mathfrak{F}(\mathfrak{r})+\frac{\lambda}{\mathfrak{y}_{2}-\mathfrak{y}_{1}}\left[\left(\mathfrak{r}-\mathfrak{y}_{1}\right) \mathfrak{F}\left(\mathfrak{y}_{1}\right)+\left(\mathfrak{y}_{2}-\mathfrak{r}\right) \mathfrak{F}\left(\mathfrak{y}_{2}\right)\right] \\
& -\frac{1}{\mathfrak{y}_{2}-\mathfrak{y}_{1}}\left[\int_{\mathfrak{y}_{1}}^{\mathfrak{r}} \mathfrak{F}(t) \mathfrak{y}_{1} d_{q} t+\int_{\mathfrak{r}}^{\mathfrak{y}_{2}} \mathfrak{F}(t)^{\mathfrak{y}_{2}} d_{q} t\right] \\
& =\frac{\left(\mathfrak{r}-\mathfrak{y}_{1}\right)^{2}}{\mathfrak{y}_{2}-\mathfrak{y}_{1}} \int_{0}^{1}(q t-\lambda)_{\mathfrak{y}_{1}} D_{q} \mathfrak{F}\left(t \mathfrak{r}+(1-t) \mathfrak{y}_{1}\right) d_{q} t \\
& -\frac{\left(\mathfrak{y}_{2}-\mathfrak{r}\right)^{2}}{\mathfrak{y}_{2}-\mathfrak{y} 1} \int_{0}^{1}(q t-\lambda)^{\mathfrak{y}_{2}} D_{q} \mathfrak{F}\left(t \mathfrak{r}+(1-t) \mathfrak{y}_{2}\right) d_{q} t .
\end{aligned}
$$


Proof. From Lemma 1 and Definition 2, we have

$$
\begin{aligned}
& \int_{0}^{1}(q t-\lambda)_{\mathfrak{y}_{1}} D_{q} \mathfrak{F}\left(t \mathfrak{r}+(1-t) \mathfrak{y}_{1}\right) d_{q} t \\
= & \left.\frac{(q t-\lambda) \mathfrak{F}\left(t \mathfrak{r}+(1-t) \mathfrak{y}_{1}\right)}{\mathfrak{r}-\mathfrak{y}_{1}}\right|_{0} ^{1}-\frac{q}{\mathfrak{r}-\mathfrak{y}_{1}} \int_{0}^{1} \mathfrak{F}\left(q \mathfrak{t}+(1-q t) \mathfrak{y}_{1}\right) d_{q} t \\
= & \frac{(q-\lambda) \mathfrak{F}(\mathfrak{r})+\lambda \mathfrak{F}\left(\mathfrak{y}_{1}\right)}{\mathfrak{r}-\mathfrak{y}_{1}}-\frac{1}{\left(\mathfrak{r}-\mathfrak{y}_{1}\right)^{2}} \int_{\mathfrak{y}_{1}}^{\mathfrak{r}} \mathfrak{F}(t)_{\mathfrak{y}_{1}} d_{q} t+\frac{1-q}{\mathfrak{r}-\mathfrak{y}_{1}} \mathfrak{F}(\mathfrak{r}) \\
= & \frac{1-\lambda}{\mathfrak{r}-\mathfrak{y}_{1}} \mathfrak{F}(\mathfrak{r})+\frac{\lambda \mathfrak{F}\left(\mathfrak{y}_{1}\right)}{\mathfrak{r}-\mathfrak{y}_{1}}-\frac{1}{\left(\mathfrak{r}-\mathfrak{y}_{1}\right)^{2}} \int_{\mathfrak{y}_{1}}^{\mathfrak{r}} \mathfrak{F}(t)_{\mathfrak{y}_{1}} d_{q} t .
\end{aligned}
$$

Now from Lemma 2 and Definition 3, we have

$$
\begin{aligned}
& \int_{0}^{1}(q t-\lambda){ }^{\mathfrak{y}_{2}} D_{q} \mathfrak{F}\left(t \mathfrak{r}+(1-t) \mathfrak{y}_{2}\right) d_{q} t \\
= & \frac{1}{\left(\mathfrak{y}_{2}-\mathfrak{r}\right)^{2}} \int_{\mathfrak{r}}^{\mathfrak{y}_{2}} \mathfrak{F}(t)^{\mathfrak{y}_{2}} d_{q} t-\frac{1-\lambda}{\mathfrak{y}_{2}-\mathfrak{r}} \mathfrak{F}(\mathfrak{r})-\frac{\lambda \mathfrak{F}\left(\mathfrak{y}_{2}\right)}{\mathfrak{y}_{2}-\mathfrak{r}} .
\end{aligned}
$$

Thus, we obtain the required equality (10) by subtracting (12) from (11) after multiplying $\frac{\left(\mathfrak{r}-\mathfrak{y}_{1}\right)^{2}}{\mathfrak{y}_{2}-\mathfrak{y}_{1}}$ and $\frac{\left(\mathfrak{y}_{2}-\mathfrak{r}\right)^{2}}{\mathfrak{y}_{2}-\mathfrak{y}_{1}}$ with (11) and (12), respectively.

Remark 2. In Lemma 3, we have

(i) If we set $\lambda=0$, then we obtain the following quantum integral identity:

$$
\begin{aligned}
\mathfrak{F}(\mathfrak{r})-\frac{1}{\mathfrak{y}_{2}-\mathfrak{y}_{1}}\left[\int_{\mathfrak{y}_{1}}^{\mathfrak{r}} \mathfrak{F}(t) \mathfrak{y}_{1} d_{q} t+\int_{\mathfrak{r}}^{\mathfrak{y}_{2}} \mathfrak{F}(t) \mathfrak{y}^{\mathfrak{y}_{2}} d_{q} t\right] \\
=\frac{\left(\mathfrak{r}-\mathfrak{y}_{1}\right)^{2}}{\mathfrak{y}_{2}-\mathfrak{y}_{1}} \int_{0}^{1} q t_{\mathfrak{y}_{1}} D_{q} \mathfrak{F}\left(\mathfrak{t r}+(1-t) \mathfrak{y}_{1}\right) d_{q} t \\
\quad-\frac{\left(\mathfrak{y}_{2}-\mathfrak{r}\right)^{2}}{\mathfrak{y}_{2}-\mathfrak{y}_{1}} \int_{0}^{1} q t^{\mathfrak{y}_{2}} D_{q} \mathfrak{F}\left(\mathfrak{t r}+(1-t) \mathfrak{y}_{2}\right) d_{q} t
\end{aligned}
$$

which is proved by Budak et al. in [2].

(ii) If we set $\lambda=1$ and $q \rightarrow 1^{-}$, then we recapture [28] [Lemma 1].

(iii) If we set $\lambda=0$ and $q \rightarrow 1^{-}$, then we recapture [27] [Lemma 1].

Corollary 1. In Lemma 3, we have

(i) If we set $\lambda=0$ and $\mathfrak{r}=\frac{\mathfrak{y}_{1}+\mathfrak{y}_{2}}{2}$, then we obtain the following equality:

$$
\begin{aligned}
\mathfrak{F}\left(\frac{\mathfrak{y}_{1}+\mathfrak{y}_{2}}{2}\right)-\frac{1}{\mathfrak{y}_{2}-\mathfrak{y}_{1}}\left[\int_{\mathfrak{y}_{1}}^{\frac{\mathfrak{y}_{1}+\mathfrak{y}_{2}}{2}} \mathfrak{F}(t)_{\mathfrak{y}_{1}} d_{q} t+\int_{\frac{\mathfrak{y}_{1}+\mathfrak{y}_{2}}{2}}^{\mathfrak{y}_{2}} \mathfrak{F}(t)^{\mathfrak{y}_{2}} d_{q} t\right] \\
=\frac{\mathfrak{y}_{2}-\mathfrak{y}_{1}}{4}\left[\int_{0}^{1} q t_{\mathfrak{y}_{1}} D_{q} \mathfrak{F}\left(t\left(\frac{\mathfrak{y}_{1}+\mathfrak{y}_{2}}{2}\right)+(1-t) \mathfrak{y}_{1}\right) d_{q} t\right. \\
\left.\quad-\int_{0}^{1} q t^{\mathfrak{y}_{2}} D_{q} \mathfrak{F}\left(t\left(\frac{\mathfrak{y}_{1}+\mathfrak{y}_{2}}{2}\right)+(1-t) \mathfrak{y}_{2}\right) d_{q} t\right] .
\end{aligned}
$$


(ii) If we set $\lambda=1$ and $\mathfrak{r}=\frac{\mathfrak{y}_{1}+\mathfrak{y}_{2}}{2}$, then we obtain the following equality:

$$
\begin{aligned}
& \frac{\mathfrak{F}\left(\mathfrak{y}_{1}\right)+\mathfrak{F}\left(\mathfrak{y}_{2}\right)}{2}-\frac{1}{\mathfrak{y}_{2}-\mathfrak{y}_{1}}\left[\int_{\mathfrak{y}_{1}}^{\frac{\mathfrak{y}_{1}+\mathfrak{y}_{2}}{2}} \mathfrak{F}(t)_{\mathfrak{y}_{1}} d_{q} t+\int_{\frac{\mathfrak{y}_{1}+\mathfrak{y}_{2}}{2}}^{\mathfrak{y}_{2}} \mathfrak{F}(t)^{\mathfrak{y}_{2}} d_{q} t\right] \\
& =\frac{\mathfrak{y}_{2}-\mathfrak{y}_{1}}{4}\left[\int_{0}^{1}(q t-1) \mathfrak{y}_{1} D_{q} \mathfrak{F}\left(t\left(\frac{\mathfrak{y}_{1}+\mathfrak{y}_{2}}{2}\right)+(1-t) \mathfrak{y}_{1}\right) d_{q} t\right. \\
& \left.\quad-\int_{0}^{1}(q t-1)^{\mathfrak{y}_{2}} D_{q} \mathfrak{F}\left(t\left(\frac{\mathfrak{y}_{1}+\mathfrak{y}_{2}}{2}\right)+(1-t) \mathfrak{y}_{2}\right) d_{q} t\right] .
\end{aligned}
$$

\section{Main Results}

In this section, we prove different versions of quantum integral inequalities for differentiable convex and bounded functions.

Theorem 7. Under the assumption of Lemma 3, if $\left|\mathfrak{y}_{1} D_{q} \mathfrak{F}\right|$ and $\left|\mathfrak{y}_{2} D_{q} \mathfrak{F}\right|$ are convex mappings over $\left[\mathfrak{y}_{1}, \mathfrak{y}_{2}\right]$, then we have the following inequality:

$$
\begin{aligned}
& \mid(1-\lambda) \mathfrak{F}(\mathfrak{r})+\frac{\lambda}{\mathfrak{y}_{2}-\mathfrak{y}_{1}}\left[\left(\mathfrak{r}-\mathfrak{y}_{1}\right) \mathfrak{F}\left(\mathfrak{y}_{1}\right)+\left(\mathfrak{y}_{2}-\mathfrak{r}\right) \mathfrak{F}\left(\mathfrak{y}_{2}\right)\right] \\
& \left.-\frac{1}{\mathfrak{y}_{2}-\mathfrak{y}_{1}}\left[\int_{\mathfrak{y}_{1}}^{\mathfrak{r}} \mathfrak{F}(t) \mathfrak{y}_{1} d_{q} t+\int_{\mathfrak{r}}^{\mathfrak{y}_{2}} \mathfrak{F}(t)\right)^{\mathfrak{y}_{2}} d_{q} t\right] \mid \\
& \leq \frac{\left(\mathfrak{r}-\mathfrak{y}_{1}\right)^{2}}{\mathfrak{y}_{2}-\mathfrak{y}_{1}}\left(\left.\Delta_{1}(\lambda ; q)\right|_{\mathfrak{y}_{1}} D_{q} \mathfrak{F}(\mathfrak{r})\left|+\Delta_{2}(\lambda ; q)\right| \mathfrak{y}_{1} D_{q} \mathfrak{F}\left(\mathfrak{y}_{1}\right) \mid\right) \\
& \quad+\frac{\left(\mathfrak{y}_{2}-\mathfrak{r}\right)^{2}}{\mathfrak{y}_{2}-\mathfrak{y}_{1}}\left(\left.\Delta_{1}(\lambda ; q)\right|^{\mathfrak{y}_{2}} D_{q} \mathfrak{F}(\mathfrak{r})\left|+\Delta_{2}(\lambda ; q)\right|^{\mathfrak{y}_{2}} D_{q} \mathfrak{F}\left(\mathfrak{y}_{2}\right) \mid\right),
\end{aligned}
$$

where

$$
\begin{aligned}
\Delta_{1}(\lambda ; q) & =\int_{0}^{1}|q t-\lambda| t d_{q} t \\
& =\left\{\begin{array}{c}
\frac{\lambda[3]_{q}-q[2]_{q}}{[2]_{q}[3]_{q}}, 0<q<\lambda ; \\
\frac{2 \lambda^{3}+q[2]_{q}-\lambda[3]_{q}}{[2]_{q}[3]_{q}}, \lambda \leq q<1
\end{array}\right.
\end{aligned}
$$

and

$$
\begin{aligned}
\Delta_{2}(\lambda ; q) & =\int_{0}^{1}|q t-\lambda|(1-t) d_{q} t \\
& =\left\{\begin{array}{c}
\frac{\lambda[2]_{q}-q}{[2]_{q}}-\frac{\lambda[3]_{q}-q[2]_{q}}{[2]_{q}[3]_{q}}, 0<q<\lambda ; \\
\frac{2 \lambda^{2}+q-\lambda[2]_{q}}{[2]_{q}}-\frac{2 \lambda^{3}+q[2]_{q}-\lambda[3]_{q}}{[2]_{q}[3]_{q}}, \lambda \leq q<1 .
\end{array}\right.
\end{aligned}
$$


Proof. By taking the modulus in (10) and using the convexity of $\left|\mathfrak{y}_{1} D_{q} \mathfrak{F}\right|$ and $\left|\mathfrak{y}_{2} D_{q} \mathfrak{F}\right|$, we have

$$
\begin{aligned}
& \mid(1-\lambda) \mathfrak{F}(\mathfrak{r})+\frac{\lambda}{\mathfrak{y}_{2}-\mathfrak{y}_{1}}\left[\left(\mathfrak{r}-\mathfrak{y}_{1}\right) \mathfrak{F}\left(\mathfrak{y}_{1}\right)+\left(\mathfrak{y}_{2}-\mathfrak{r}\right) \mathfrak{F}\left(\mathfrak{y}_{2}\right)\right] \\
& -\frac{1}{\mathfrak{y}_{2}-\mathfrak{y}_{1}}\left[\int_{\mathfrak{y}_{1}}^{\mathfrak{r}} \mathfrak{F}(t) \mathfrak{y}_{1} d_{q} t+\int_{\mathfrak{r}}^{\mathfrak{y}_{2}} \mathfrak{F}(t)^{\mathfrak{y}_{2}} d_{q} t\right] \mid \\
& \leq \frac{\left(\mathfrak{r}-\mathfrak{y}_{1}\right)^{2}}{\mathfrak{y}_{2}-\mathfrak{y}_{1}} \int_{0}^{1}|q t-\lambda|\left|\mathfrak{y}_{1} D_{q} \mathfrak{F}\left(t \mathfrak{r}+(1-t) \mathfrak{y}_{1}\right)\right| d_{q} t \\
& \quad+\frac{\left(\mathfrak{y}_{2}-\mathfrak{r}\right)^{2}}{\mathfrak{y}_{2}-\mathfrak{y}_{1}} \int_{0}^{1}|q t-\lambda|\left|\mathfrak{y}_{2} D_{q} \mathfrak{F}\left(\mathfrak{t r}+(1-t) \mathfrak{y}_{2}\right)\right| d_{q} t \\
& \leq \frac{\left(\mathfrak{r}-\mathfrak{y}_{1}\right)^{2}}{\mathfrak{y}_{2}-\mathfrak{y}_{1}} \int_{0}^{1}|q t-\lambda|\left[t\left|\mathfrak{y}_{1} D_{q} \mathfrak{F}(\mathfrak{r})\right|+(1-t)\left|\mathfrak{y}_{1} D_{q} \mathfrak{F}\left(\mathfrak{y}_{1}\right)\right|\right] d_{q} t \\
& +\frac{\left(\mathfrak{y}_{2}-\mathfrak{r}\right)^{2}}{\mathfrak{y}_{2}-\mathfrak{y}_{1}} \int_{0}^{1}|q t-\lambda|\left[t\left|\mathfrak{y}_{2} D_{q} \mathfrak{F}(\mathfrak{r})\right|+(1-t)\left|\mathfrak{y}_{2} D_{q} \mathfrak{F}\left(\mathfrak{y}_{2}\right)\right|\right] d_{q} t \\
& =\frac{\left(\mathfrak{r}-\mathfrak{y}_{1}\right)^{2}}{\mathfrak{y}_{2}-\mathfrak{y}_{1}}\left(\left.\Delta_{1}(\lambda ; q)\right|_{\mathfrak{y}_{1}} D_{q} \mathfrak{F}(\mathfrak{r})\left|+\Delta_{2}(\lambda ; q)\right|_{\mathfrak{y}_{1}} D_{q} \mathfrak{F}\left(\mathfrak{y}_{1}\right) \mid\right) \\
& +\frac{\left(\mathfrak{y}_{2}-\mathfrak{r}\right)^{2}}{\mathfrak{y}_{2}-\mathfrak{y}_{1}}\left(\left.\Delta_{1}(\lambda ; q)\right|^{\mathfrak{y}_{2}} D_{q} \mathfrak{F}(\mathfrak{r})\left|+\Delta_{2}(\lambda ; q)\right|^{\mathfrak{y}_{2}} D_{q} \mathfrak{F}\left(\mathfrak{y}_{2}\right) \mid\right) .
\end{aligned}
$$

Thus, the proof is completed.

Remark 3. In Theorem 7, we have

(i) If we set $\lambda=0$, then we obtain the following quantum Ostrowski type integral inequality:

$$
\begin{aligned}
& \left|\mathfrak{F}(\mathfrak{r})-\frac{1}{\mathfrak{y}_{2}-\mathfrak{y}_{1}}\left[\int_{\mathfrak{y}_{1}}^{\mathfrak{r}} \mathfrak{F}(t) \mathfrak{y}_{1} d_{q} t+\int_{\mathfrak{r}}^{\mathfrak{y}_{2}} \mathfrak{F}(t){ }^{\mathfrak{y}_{2}} d_{q} t\right]\right| \\
& \leq \frac{q}{\left(\mathfrak{y}_{2}-\mathfrak{y}_{1}\right)[2]_{q}[3]_{q}}\left[\left(\mathfrak{r}-\mathfrak{y}_{1}\right)^{2}\left([2]_{q}\left|\mathfrak{y}_{1} D_{q} \mathfrak{F}(\mathfrak{r})\right|+q^{2}\left|\mathfrak{y}_{1} D_{q} \mathfrak{F}\left(\mathfrak{y}_{1}\right)\right|\right)\right. \\
& \left.\quad+\left(\mathfrak{y}_{2}-\mathfrak{r}\right)^{2}\left([2]_{q}\left|{ }^{\mathfrak{y}_{2}} D_{q} \mathfrak{F}(\mathfrak{r})\right|+q^{2}\left|\mathfrak{y}_{2} D_{q} \mathfrak{F}\left(\mathfrak{y}_{2}\right)\right|\right)\right],
\end{aligned}
$$

which is proved by Budak et al. in [2].

(ii) If we set $\lambda=1$ and $q \rightarrow 1^{-}$, then we recapture [28] [Theorem 4].

Corollary 2. In Theorem 7 , we have

(i) If we assume that ${ }_{\mathfrak{y}_{1}} D_{q} \mathfrak{F}(\mathfrak{r}),{ }^{\mathfrak{y}_{2}} D_{q} \mathfrak{F}(\mathfrak{r}) \leq M$, then we obtain the following new inequality:

$$
\begin{aligned}
& \mid(1-\lambda) \mathfrak{F}(\mathfrak{r})+\frac{\lambda}{\mathfrak{y}_{2}-\mathfrak{y}_{1}}\left[\left(\mathfrak{r}-\mathfrak{y}_{1}\right) \mathfrak{F}\left(\mathfrak{y}_{1}\right)+\left(\mathfrak{y}_{2}-\mathfrak{r}\right) \mathfrak{F}\left(\mathfrak{y}_{2}\right)\right] \\
& -\frac{1}{\mathfrak{y}_{2}-\mathfrak{y}_{1}}\left[\int_{\mathfrak{y}_{1}}^{\mathfrak{r}} \mathfrak{F}(t) \mathfrak{y}_{1} d_{q} t+\int_{\mathfrak{r}}^{\mathfrak{y}_{2}} \mathfrak{F}(t)^{\mathfrak{y}_{2}} d_{q} t\right] \mid \\
& \leq \frac{M}{\mathfrak{y}_{2}-\mathfrak{y}_{1}}\left[\left(\Delta_{1}(\lambda ; q)+\Delta_{2}(\lambda ; q)\right)\left(\left(\mathfrak{r}-\mathfrak{y}_{1}\right)^{2}+\left(\mathfrak{y}_{2}-\mathfrak{r}\right)^{2}\right)\right] .
\end{aligned}
$$


(ii) If we set $\lambda=0$ and $\mathfrak{r}=\frac{\mathfrak{y}_{1}+\mathfrak{y}_{2}}{2}$, then we obtain the following quantum midpoint type inequality:

$$
\begin{aligned}
& \left|\mathfrak{F}\left(\frac{\mathfrak{y}_{1}+\mathfrak{y}_{2}}{2}\right)-\frac{1}{\mathfrak{y}_{2}-\mathfrak{y}_{1}}\left[\int_{\mathfrak{y}_{1}}^{\frac{\mathfrak{y}_{1}+\mathfrak{y}_{2}}{2}} \mathfrak{F}(t)_{\mathfrak{y}_{1}} d_{q} t+\int_{\frac{\mathfrak{y}_{1}+\mathfrak{y}_{2}}{2}}^{\mathfrak{y}_{2}} \mathfrak{F}(t)^{\mathfrak{y}_{2}} d_{q} t\right]\right| \\
& \leq \frac{q\left(\mathfrak{y}_{2}-\mathfrak{y}_{1}\right)}{4[2]_{q}[3]_{q}}\left[\left([2]_{q}\left|\mathfrak{y}_{1} D_{q} \mathfrak{F}\left(\frac{\mathfrak{y}_{1}+\mathfrak{y}_{2}}{2}\right)\right|+q^{2}\left|\mathfrak{y}_{1} D_{q} \mathfrak{F}\left(\mathfrak{y}_{1}\right)\right|\right)\right. \\
& \left.\quad+\left([2]_{q}\left|\mathfrak{y}_{2} D_{q} \mathfrak{F}\left(\frac{\mathfrak{y}_{1}+\mathfrak{y}_{2}}{2}\right)\right|+q^{2}\left|\mathfrak{y}_{2} D_{q} \mathfrak{F}\left(\mathfrak{y}_{2}\right)\right|\right)\right] .
\end{aligned}
$$

(iii) If we set $\lambda=1$ and $\mathfrak{r}=\frac{\mathfrak{y}_{1}+\mathfrak{y}_{2}}{2}$, then we obtain the following quantum trapezoidal type inequality:

$$
\begin{aligned}
& \left.\left|\frac{\mathfrak{F}\left(\mathfrak{y}_{1}\right)+\mathfrak{F}\left(\mathfrak{y}_{2}\right)}{2}-\frac{1}{\mathfrak{y}_{2}-\mathfrak{y}_{1}}\left[\int_{\mathfrak{y}_{1}}^{\frac{\mathfrak{y}_{1}+\mathfrak{y}_{2}}{2}} \mathfrak{F}(t)_{\mathfrak{y}_{1}} d_{q} t+\int_{\frac{\mathfrak{y}_{1}+\mathfrak{y}_{2}}{2}}^{\mathfrak{y}_{2}} \mathfrak{F}(t)^{\mathfrak{y}_{2}} d_{q} t\right]\right|+\left.\Delta_{2}(1 ; q)\right|_{\mathfrak{y}_{1}} D_{q} \mathfrak{F}\left(\mathfrak{y}_{1}\right) \mid\right) \\
& \leq \frac{\left(\mathfrak{y}_{2}-\mathfrak{y}_{1}\right)}{4}\left[\left(\Delta_{1}(1 ; q)\left|\mathfrak{y}_{1} D_{q} \mathfrak{F}\left(\frac{\mathfrak{y}_{1}+\mathfrak{y}_{2}}{2}\right)\right|\right.\right. \\
& \left.\quad+\left(\Delta_{1}(1 ; q)\left|\mathfrak{y}_{2} D_{q} \mathfrak{F}\left(\frac{\mathfrak{y}_{1}+\mathfrak{y}_{2}}{2}\right)\right|+\left.\Delta_{2}(1 ; q)\right|^{\mathfrak{y}_{2}} D_{q} \mathfrak{F}\left(\mathfrak{y}_{2}\right) \mid\right)\right] .
\end{aligned}
$$

(iv) If we set $\lambda=\frac{1}{3}$ and $\mathfrak{r}=\frac{\mathfrak{y}_{1}+\mathfrak{y}_{2}}{2}$, then we obtain the following quantum Simpson's type inequality:

$$
\begin{aligned}
& \left|\frac{1}{6}\left[\mathfrak{F}\left(\mathfrak{y}_{1}\right)+4 \mathfrak{F}\left(\frac{\mathfrak{y}_{1}+\mathfrak{y}_{2}}{2}\right)+\mathfrak{F}\left(\mathfrak{y}_{2}\right)\right]-\frac{1}{\mathfrak{y}_{2}-\mathfrak{y}_{1}}\left[\int_{\mathfrak{y}_{1}}^{\frac{\mathfrak{y}_{1}+\mathfrak{y}_{2}}{2}} \mathfrak{F}(t) \mathfrak{y}_{1} d_{q} t+\int_{\frac{\mathfrak{y}_{1}+\mathfrak{y}_{2}}{2}}^{\mathfrak{y}_{2}} \mathfrak{F}(t)^{\mathfrak{y}_{2}} d_{q} t\right]\right| \\
& \leq \frac{\left(\mathfrak{y}_{2}-\mathfrak{y}_{1}\right)}{4}\left[\left(\Delta_{1}\left(\frac{1}{3} ; q\right)\left|\mathfrak{y}_{1} D_{q} \mathfrak{F}\left(\frac{\mathfrak{y}_{1}+\mathfrak{y}_{2}}{2}\right)\right|+\Delta_{2}\left(\frac{1}{3} ; q\right)\left|\mathfrak{y}_{1} D_{q} \mathfrak{F}\left(\mathfrak{y}_{1}\right)\right|\right)\right. \\
& \left.\quad+\left(\Delta_{1}\left(\frac{1}{3} ; q\right)\left|\mathfrak{y}_{2} D_{q} \mathfrak{F}\left(\frac{\mathfrak{y}_{1}+\mathfrak{y}_{2}}{2}\right)\right|+\Delta_{2}\left(\frac{1}{3} ; q\right)\left|{ }^{\mathfrak{y}_{2}} D_{q} \mathfrak{F}\left(\mathfrak{y}_{2}\right)\right|\right)\right] .
\end{aligned}
$$

Theorem 8. Under the assumption of Lemma 3, if $\left|\mathfrak{y}_{1} D_{q} \mathfrak{F}\right|^{s},\left|\mathfrak{y}_{2} D_{q} \mathfrak{F}\right|^{s}$, where $s \geq 1$ are convex mappings over $\left[\mathfrak{y}_{1}, \mathfrak{y}_{2}\right]$, then we have the following quantum integral inequality:

$$
\begin{aligned}
& \mid(1-\lambda) \mathfrak{F}(\mathfrak{r})+\frac{\lambda}{\mathfrak{y}_{2}-\mathfrak{y}_{1}}\left[\left(\mathfrak{r}-\mathfrak{y}_{1}\right) \mathfrak{F}\left(\mathfrak{y}_{1}\right)+\left(\mathfrak{y}_{2}-\mathfrak{r}\right) \mathfrak{F}\left(\mathfrak{y}_{2}\right)\right] \\
& -\frac{1}{\mathfrak{y}_{2}-\mathfrak{y}_{1}}\left[\int_{\mathfrak{y}_{1}}^{\mathfrak{r}} \mathfrak{F}(t) \mathfrak{y}_{1} d_{q} t+\int_{\mathfrak{r}}^{\mathfrak{y}_{2}} \mathfrak{F}(t) \mathfrak{y}_{2} d_{q} t\right] \mid \\
& \leq \Delta_{5}(\lambda ; q)^{1-\frac{1}{s}}\left[\frac{\left(\mathfrak{r}-\mathfrak{y}_{1}\right)^{2}}{\mathfrak{y}_{2}-\mathfrak{y}_{1}}\left(\left.\left.\Delta_{1}(\lambda ; q)\right|_{\mathfrak{y}_{1}} D_{q} \mathfrak{F}(\mathfrak{r})\right|^{s}+\left.\left.\Delta_{2}(\lambda ; q)\right|_{\mathfrak{y}_{1}} D_{q} \mathfrak{F}\left(\mathfrak{y}_{1}\right)\right|^{s}\right)^{\frac{1}{s}}\right. \\
& \left.\quad+\frac{\left(\mathfrak{y}_{2}-\mathfrak{r}\right)^{2}}{\mathfrak{y}_{2}-\mathfrak{y}_{1}}\left(\left.\left.\Delta_{1}(\lambda ; q)\right|^{\mathfrak{y}_{2}} D_{q} \mathfrak{F}(\mathfrak{r})\right|^{s}+\left.\left.\Delta_{2}(\lambda ; q)\right|^{\mathfrak{y}_{2}} D_{q} \mathfrak{F}\left(\mathfrak{y}_{2}\right)\right|^{s}\right)^{\frac{1}{s}}\right],
\end{aligned}
$$

where $\Delta_{i}(\lambda ; q), i=1,2$ are defined in Theorem 7 and

$$
\Delta_{5}(\lambda ; q)=\int_{0}^{1}|q t-\lambda| d_{q} t=\left\{\begin{array}{c}
\frac{\lambda[2]_{q}-q}{[2]_{q}}, 0<q<\lambda \\
\frac{2 \lambda^{2}+q-\lambda[2]_{q}}{[2]_{q}}, \quad \lambda \leq q<1 .
\end{array}\right.
$$


Proof. By taking modulus in (10) and using power mean inequality, we have

$$
\begin{aligned}
& \mid(1-\lambda) \mathfrak{F}(\mathfrak{r})+\frac{\lambda}{\mathfrak{y}_{2}-\mathfrak{y}_{1}}\left[\left(\mathfrak{r}-\mathfrak{y}_{1}\right) \mathfrak{F}\left(\mathfrak{y}_{1}\right)+\left(\mathfrak{y}_{2}-\mathfrak{r}\right) \mathfrak{F}\left(\mathfrak{y}_{2}\right)\right] \\
& -\frac{1}{\mathfrak{y}_{2}-\mathfrak{y}_{1}}\left[\int_{\mathfrak{y}_{1}}^{\mathfrak{r}} \mathfrak{F}(t) \mathfrak{y}_{1} d_{q} t+\int_{\mathfrak{r}}^{\mathfrak{y}_{2}} \mathfrak{F}(t)^{\mathfrak{y}_{2}} d_{q} t\right] \mid \\
& \leq \frac{\left(\mathfrak{r}-\mathfrak{y}_{1}\right)^{2}}{\mathfrak{y}_{2}-\mathfrak{y}_{1}} \int_{0}^{1}|q t-\lambda|\left|\mathfrak{y}_{1} D_{q} \mathfrak{F}\left(\mathfrak{t r}+(1-t) \mathfrak{y}_{1}\right)\right| d_{q} t \\
& +\frac{\left(\mathfrak{y}_{2}-\mathfrak{r}\right)^{2}}{\mathfrak{y}_{2}-\mathfrak{y}_{1}} \int_{0}^{1}|q t-\lambda|\left|\mathfrak{y}_{2} D_{q} \mathfrak{F}\left(t \mathfrak{r}+(1-t) \mathfrak{y}_{2}\right)\right| d_{q} t \\
& \leq \frac{\left(\mathfrak{r}-\mathfrak{y}_{1}\right)^{2}}{\mathfrak{y}_{2}-\mathfrak{y}_{1}}\left(\int_{0}^{1}|q t-\lambda| d_{q} t\right)^{1-\frac{1}{s}}\left(\int_{0}^{1}|q t-\lambda|\left|\mathfrak{y}_{1} D_{q} \mathfrak{F}\left(\mathfrak{t r}+(1-t) \mathfrak{y}_{1}\right)\right|^{s} d_{q} t\right)^{\frac{1}{s}} \\
& +\frac{\left(\mathfrak{y}_{2}-\mathfrak{r}\right)^{2}}{\mathfrak{y}_{2}-\mathfrak{y}_{1}}\left(\int_{0}^{1}|q t-\lambda| d_{q} t\right)^{1-\frac{1}{s}}\left(\int_{0}^{1}|q t-\lambda|\left|\mathfrak{y}_{2} D_{q} \mathfrak{F}\left(t \mathfrak{r}+(1-t) \mathfrak{y}_{2}\right)\right|^{s} d_{q} t\right)^{\frac{1}{s}} .
\end{aligned}
$$

Now by applying convexity, we have

$$
\begin{aligned}
& \mid(1-\lambda) \mathfrak{F}(\mathfrak{r})+\frac{\lambda}{\mathfrak{y}_{2}-\mathfrak{y}_{1}}\left[\left(\mathfrak{r}-\mathfrak{y}_{1}\right) \mathfrak{F}\left(\mathfrak{y}_{1}\right)+\left(\mathfrak{y}_{2}-\mathfrak{r}\right) \mathfrak{F}\left(\mathfrak{y}_{2}\right)\right] \\
& -\frac{1}{\mathfrak{y}_{2}-\mathfrak{y}_{1}}\left[\int_{\mathfrak{y}_{1}}^{\mathfrak{r}} \mathfrak{F}(t) \mathfrak{y}_{1} d_{q} t+\int_{\mathfrak{r}}^{\mathfrak{y}_{2}} \mathfrak{F}(t) \mathfrak{y}_{2} d_{q} t\right] \mid \\
& \leq \frac{\left(\mathfrak{r}-\mathfrak{y}_{1}\right)^{2}}{\mathfrak{y}_{2}-\mathfrak{y}_{1}}\left(\int_{0}^{1}|q t-\lambda| d_{q} t\right)^{1-\frac{1}{s}} \\
& \times\left(\int_{0}^{1}|q t-\lambda|\left[\left.\left.t\right|_{\mathfrak{y}_{1}} D_{q} \mathfrak{F}(\mathfrak{r})\right|^{s}+(1-t)\left|\mathfrak{y}_{1} D_{q} \mathfrak{F}\left(\mathfrak{y}_{1}\right)\right|^{s}\right] d_{q} t\right)^{\frac{1}{s}} \\
& +\frac{\left(\mathfrak{y}_{2}-\mathfrak{r}\right)^{2}}{\mathfrak{y}_{2}-\mathfrak{y}_{1}}\left(\int_{0}^{1}|q t-\lambda| d_{q} t\right)^{1-\frac{1}{s}} \\
& \times\left(\int_{0}^{1}|q t-\lambda|\left[t\left|\mathfrak{y}_{2} D_{q} \mathfrak{F}(\mathfrak{r})\right|^{s}+(1-t)\left|\mathfrak{y}_{2} D_{q} \mathfrak{F}\left(\mathfrak{y}_{2}\right)\right|^{s}\right] d_{q} t\right)^{\frac{1}{s}} \\
& =\frac{\left(\mathfrak{r}-\mathfrak{y}_{1}\right)^{2}}{\mathfrak{y}_{2}-\mathfrak{y}_{1}} \Delta_{5}(\lambda ; q)^{1-\frac{1}{s}}\left(\left.\left.\Delta_{1}(\lambda ; q)\right|_{\mathfrak{y}_{1}} D_{q} \mathfrak{F}(\mathfrak{r})\right|^{s}+\left.\left.\Delta_{2}(\lambda ; q)\right|_{\mathfrak{y}_{1}} D_{q} \mathfrak{F}\left(\mathfrak{y}_{1}\right)\right|^{s}\right)^{\frac{1}{s}} \\
& +\frac{\left(\mathfrak{y}_{2}-\mathfrak{r}\right)^{2}}{\mathfrak{y}_{2}-\mathfrak{y}_{1}} \Delta_{5}(\lambda ; q)^{1-\frac{1}{s}}\left(\left.\left.\Delta_{1}(\lambda ; q)\right|^{\mathfrak{y}_{2}} D_{q} \mathfrak{F}(\mathfrak{r})\right|^{s}+\left.\left.\Delta_{2}(\lambda ; q)\right|^{\mathfrak{y}_{2}} D_{q} \mathfrak{F}\left(\mathfrak{y}_{2}\right)\right|^{s}\right)^{\frac{1}{s}} .
\end{aligned}
$$

Thus, the proof is completed.

Remark 4. In Theorem 8, we have:

(i) If we set $\lambda=0$, then we obtain the following quantum Ostrowski type integral inequality:

$$
\begin{aligned}
& \left|\mathfrak{F}(\mathfrak{r})-\frac{1}{\mathfrak{y}_{2}-\mathfrak{y}_{1}}\left[\int_{\mathfrak{y}_{1}}^{\mathfrak{r}} \mathfrak{F}(t)_{\mathfrak{y}_{1}} d_{q} t+\int_{\mathfrak{r}}^{\mathfrak{y}_{2}} \mathfrak{F}(t)^{\mathfrak{y}_{2}} d_{q} t\right]\right| \\
& \leq \frac{q}{\left(\mathfrak{y}_{2}-\mathfrak{y}_{1}\right)[2]_{q}}\left[\left(\mathfrak{r}-\mathfrak{y}_{1}\right)^{2}\left(\frac{[2]_{q}\left|\mathfrak{y}_{1} D_{q} \mathfrak{F}(\mathfrak{r})\right|^{s}+q^{2}\left|\mathfrak{y}_{1} D_{q} \mathfrak{F}\left(\mathfrak{y}_{1}\right)\right|^{s}}{[3]_{q}}\right)^{\frac{1}{s}}\right. \\
& \left.\quad+\left(\mathfrak{y}_{2}-\mathfrak{r}\right)^{2}\left(\frac{[2]_{q}\left|\mathfrak{y}_{2} D_{q} \mathfrak{F}(\mathfrak{r})\right|^{s}+q^{2}\left|\mathfrak{y}_{2} D_{q} \mathfrak{F}\left(\mathfrak{y}_{2}\right)\right|^{s}}{[3]_{q}}\right)^{\frac{1}{s}}\right]
\end{aligned}
$$

which is proved by Budak et al. in [2]. 
(ii) If we set $\lambda=1$ and $q \rightarrow 1^{-}$, then we recapture [28] [Theorem 7 ].

Corollary 3. In Theorem 8 , we have

(i) If we assume that $\mathfrak{y}_{1} D_{q} \mathfrak{F}(\mathfrak{r}), \mathfrak{y}_{2} D_{q} \mathfrak{F}(\mathfrak{r}) \leq M$, then we obtain the following new inequality:

$$
\begin{aligned}
& \mid(1-\lambda) \mathfrak{F}(\mathfrak{r})+\frac{\lambda}{\mathfrak{y}_{2}-\mathfrak{y}_{1}}\left[\left(\mathfrak{r}-\mathfrak{y}_{1}\right) \mathfrak{F}\left(\mathfrak{y}_{1}\right)+\left(\mathfrak{y}_{2}-\mathfrak{r}\right) \mathfrak{F}\left(\mathfrak{y}_{2}\right)\right] \\
& -\frac{1}{\mathfrak{y}_{2}-\mathfrak{y}_{1}}\left[\int_{\mathfrak{y}_{1}}^{\mathfrak{r}} \mathfrak{F}(t) \mathfrak{y}_{1} d_{q} t+\int_{\mathfrak{r}}^{\mathfrak{y}_{2}} \mathfrak{F}(t)^{\mathfrak{y}_{2}} d_{q} t\right] \mid \\
& \leq \frac{M \Delta_{5}(\lambda ; q)^{1-\frac{1}{s}}}{\mathfrak{y}_{2}-\mathfrak{y}_{1}}\left[\left(\Delta_{1}(\lambda ; q)+\Delta_{2}(\lambda ; q)\right)^{\frac{1}{s}}\left(\left(\mathfrak{r}-\mathfrak{y}_{1}\right)^{2}+\left(\mathfrak{y}_{2}-\mathfrak{r}\right)^{2}\right)\right] .
\end{aligned}
$$

(ii) If we set $\lambda=0$ and $\mathfrak{r}=\frac{\mathfrak{y}_{1}+\mathfrak{y}_{2}}{2}$, then we obtain the following quantum midpoint type inequality:

$$
\begin{aligned}
& \left|\mathfrak{F}\left(\frac{\mathfrak{y}_{1}+\mathfrak{y}_{2}}{2}\right)-\frac{1}{\mathfrak{y}_{2}-\mathfrak{y}_{1}}\left[\int_{\mathfrak{y}_{1}}^{\frac{\mathfrak{y}_{1}+\mathfrak{y}_{2}}{2}} \mathfrak{F}(t) \mathfrak{y}_{1} d_{q} t+\int_{\frac{\mathfrak{y}_{1}+\mathfrak{y}_{2}}{2}}^{\mathfrak{y}_{2}} \mathfrak{F}(t) \mathfrak{y}_{2} d_{q} t\right]\right| \\
& \leq \frac{q\left(\mathfrak{y}_{2}-\mathfrak{y}_{1}\right)}{4[2]_{q}}\left[\left(\frac{[2]_{q}\left|\mathfrak{y}_{1} D_{q} \mathfrak{F}\left(\frac{\mathfrak{y}_{1}+\mathfrak{y}_{2}}{2}\right)\right|^{s}+q^{2}\left|\mathfrak{y}_{1} D_{q} \mathfrak{F}\left(\mathfrak{y}_{1}\right)\right|^{s}}{[3]_{q}}\right)^{\frac{1}{s}}\right. \\
& \left.\quad+\left(\frac{[2]_{q}\left|\mathfrak{y}_{2} D_{q} \mathfrak{F}\left(\frac{\mathfrak{y}_{1}+\mathfrak{y}_{2}}{2}\right)\right|^{s}+q^{2}\left|\mathfrak{y}_{2} D_{q} \mathfrak{F}\left(\mathfrak{y}_{2}\right)\right|^{s}}{[3]_{q}}\right)^{\frac{1}{s}}\right] .
\end{aligned}
$$

(iii) If we set $\lambda=1$ and $\mathfrak{r}=\frac{\mathfrak{y}_{1}+\mathfrak{y}_{2}}{2}$, then we obtain the following quantum trapezoidal type inequality:

$$
\begin{aligned}
& \left|\frac{\mathfrak{F}\left(\mathfrak{y}_{1}\right)+\mathfrak{F}\left(\mathfrak{y}_{2}\right)}{2}-\frac{1}{\mathfrak{y}_{2}-\mathfrak{y}_{1}}\left[\int_{\mathfrak{y}_{1}}^{\frac{\mathfrak{y}_{1}+\mathfrak{y}_{2}}{2}} \mathfrak{F}(t)_{\mathfrak{y}_{1}} d_{q} t+\int_{\frac{\mathfrak{y}_{1}+\mathfrak{y}_{2}}{2}}^{\mathfrak{y}_{2}} \mathfrak{F}(t)^{\mathfrak{y}_{2}} d_{q} t\right]\right| \\
& \leq \frac{\left(\mathfrak{y}_{2}-\mathfrak{y}_{1}\right)}{4} \Delta_{5}(1 ; q)^{1-\frac{1}{s}}\left[\left(\left.\left.\Delta_{1}(1 ; q)\right|_{\mathfrak{y}_{1}} D_{q} \mathfrak{F}\left(\frac{\mathfrak{y}_{1}+\mathfrak{y}_{2}}{2}\right)\right|^{s}+\left.\left.\Delta_{2}(1 ; q)\right|_{\mathfrak{y}_{1}} D_{q} \mathfrak{F}\left(\mathfrak{y}_{1}\right)\right|^{s}\right)^{\frac{1}{s}}\right. \\
& \left.\quad+\left(\Delta_{1}(1 ; q)\left|\mathfrak{y}_{2} D_{q} \mathfrak{F}\left(\frac{\mathfrak{y}_{1}+\mathfrak{y}_{2}}{2}\right)\right|^{s}+\Delta_{2}(1 ; q)\left|\mathfrak{y}_{2} D_{q} \mathfrak{F}\left(\mathfrak{y}_{2}\right)\right|^{s}\right)^{\frac{1}{s}}\right]
\end{aligned}
$$

(iv) If we set $\lambda=\frac{1}{3}$ and $\mathfrak{r}=\frac{\mathfrak{y}_{1}+\mathfrak{y}_{2}}{2}$, then we obtain the following quantum Simpson's type inequality:

$$
\begin{aligned}
& \left|\frac{1}{6}\left[\mathfrak{F}\left(\mathfrak{y}_{1}\right)+4 \mathfrak{F}\left(\frac{\mathfrak{y}_{1}+\mathfrak{y}_{2}}{2}\right)+\mathfrak{F}\left(\mathfrak{y}_{2}\right)\right]-\frac{1}{\mathfrak{y}_{2}-\mathfrak{y}_{1}}\left[\int_{\mathfrak{y}_{1}}^{\frac{\mathfrak{y}_{1}+\mathfrak{y}_{2}}{2}} \mathfrak{F}(t)_{\mathfrak{y}_{1}} d_{q} t+\int_{\frac{\mathfrak{y}_{1}+\mathfrak{y}_{2}}{2}}^{\mathfrak{y}_{2}} \mathfrak{F}(t)^{\mathfrak{y}_{2}} d_{q} t\right]\right| \\
& \leq \frac{\left(\mathfrak{y}_{2}-\mathfrak{y}_{1}\right)}{4} \Delta_{5}\left(\frac{1}{3} ; q\right)^{1-\frac{1}{s}}\left[\left(\Delta_{1}\left(\frac{1}{3} ; q\right)\left|\mathfrak{y}_{1} D_{q} \mathfrak{F}\left(\frac{\mathfrak{y}_{1}+\mathfrak{y}_{2}}{2}\right)\right|^{s}+\Delta_{2}\left(\frac{1}{3} ; q\right)\left|\mathfrak{y}_{1} D_{q} \mathfrak{F}\left(\mathfrak{y}_{1}\right)\right|^{s}\right)^{\frac{1}{s}}\right. \\
& \left.\quad+\left(\Delta_{1}\left(\frac{1}{3} ; q\right)\left|\mathfrak{y}_{2} D_{q} \mathfrak{F}\left(\frac{\mathfrak{y}_{1}+\mathfrak{y}_{2}}{2}\right)\right|^{s}+\Delta_{2}\left(\frac{1}{3} ; q\right)\left|\mathfrak{y}_{2} D_{q} \mathfrak{F}\left(\mathfrak{y}_{2}\right)\right|^{s}\right)^{\frac{1}{s}}\right] .
\end{aligned}
$$


Theorem 9. Under the assumption of Lemma 3, if $s>1$ is a real number and $\left|\mathfrak{y}_{1} D_{q} \mathfrak{F}\right|^{s}$ and $\left|\mathfrak{y}_{2} D_{q} \mathfrak{F}\right|^{s}$ are convex mappings over $\left[\mathfrak{y}_{1}, \mathfrak{y}_{2}\right]$, then we have the following quantum integral inequality:

$$
\begin{aligned}
& \mid(1-\lambda) \mathfrak{F}(\mathfrak{r})+\frac{\lambda}{\mathfrak{y}_{2}-\mathfrak{y}_{1}}\left[\left(\mathfrak{r}-\mathfrak{y}_{1}\right) \mathfrak{F}\left(\mathfrak{y}_{1}\right)+\left(\mathfrak{y}_{2}-\mathfrak{r}\right) \mathfrak{F}\left(\mathfrak{y}_{2}\right)\right] \\
& -\frac{1}{\mathfrak{y}_{2}-\mathfrak{y}_{1}}\left[\int_{\mathfrak{y}_{1}}^{\mathfrak{r}} \mathfrak{F}(t) \mathfrak{y}_{1} d_{q} t+\int_{\mathfrak{r}}^{\mathfrak{y}_{2}} \mathfrak{F}(t)^{\mathfrak{y}_{2}} d_{q} t\right] \mid \\
& \leq \Delta_{7}(\lambda ; q, r)^{\frac{1}{r}}\left[\frac{\left(\mathfrak{r}-\mathfrak{y}_{1}\right)^{2}}{\mathfrak{y}_{2}-\mathfrak{y}_{1}}\left(\frac{\left|\mathfrak{y}_{1} D_{q} \mathfrak{F}(\mathfrak{r})\right|^{s}+q\left|\mathfrak{y}_{1} D_{q} \mathfrak{F}\left(\mathfrak{y}_{1}\right)\right|^{s}}{[2]_{q}}\right)^{\frac{1}{s}}\right. \\
& \left.+\frac{\left(\mathfrak{y}_{2}-\mathfrak{r}\right)^{2}}{\mathfrak{y}_{2}-\mathfrak{y}_{1}}\left(\frac{\left|\mathfrak{y}_{2} D_{q} \mathfrak{F}(\mathfrak{r})\right|^{S}+q\left|\mathfrak{y}_{2} D_{q} \mathfrak{F}\left(\mathfrak{y}_{2}\right)\right|^{S}}{[2]_{q}}\right)^{\frac{1}{s}}\right],
\end{aligned}
$$

where $s^{-1}+r^{-1}=1$ and

$$
\begin{aligned}
\Delta_{7}(\lambda ; q, r) & =\int_{0}^{1}|q t-\lambda|^{r} d_{q} t \\
& =(1-q) \sum_{n=0}^{\infty} q^{n}\left|q^{n+1}-\lambda\right|^{r} .
\end{aligned}
$$

Proof. Taking modulus in Lemma 3 and applying the Hölder's inequality, we have

$$
\begin{aligned}
& \mid(1-\lambda) \mathfrak{F}(\mathfrak{r})+\frac{\lambda}{\mathfrak{y}_{2}-\mathfrak{y}_{1}}\left[\left(\mathfrak{r}-\mathfrak{y}_{1}\right) \mathfrak{F}\left(\mathfrak{y}_{1}\right)+\left(\mathfrak{y}_{2}-\mathfrak{r}\right) \mathfrak{F}\left(\mathfrak{y}_{2}\right)\right] \\
& -\frac{1}{\mathfrak{y}_{2}-\mathfrak{y}_{1}}\left[\int_{\mathfrak{y}_{1}}^{\mathfrak{r}} \mathfrak{F}(t) \mathfrak{y}_{1} d_{q} t+\int_{\mathfrak{r}}^{\mathfrak{y}_{2}} \mathfrak{F}(t)^{\mathfrak{y}_{2}} d_{q} t\right] \mid \\
& \leq \frac{\left(\mathfrak{r}-\mathfrak{y}_{1}\right)^{2}}{\mathfrak{y}_{2}-\mathfrak{y}_{1}} \int_{0}^{1}|q t-\lambda|\left|\mathfrak{y}_{1} D_{q} \mathfrak{F}\left(\mathfrak{t r}+(1-t) \mathfrak{y}_{1}\right)\right| d_{q} t \\
& +\left.\frac{\left(\mathfrak{y}_{2}-\mathfrak{r}\right)^{2}}{\mathfrak{y}_{2}-\mathfrak{y}_{1}} \int_{0}^{1}|q t-\lambda|\right|^{\mathfrak{y}_{2}} D_{q} \mathfrak{F}\left(t \mathfrak{r}+(1-t) \mathfrak{y}_{2}\right) \mid d_{q} t \\
& \leq \frac{\left(\mathfrak{r}-\mathfrak{y}_{1}\right)^{2}}{\mathfrak{y}_{2}-\mathfrak{y}_{1}}\left(\int_{0}^{1}|q t-\lambda|^{r} d_{q} t\right)^{\frac{1}{r}}\left(\int_{0}^{1}\left|\mathfrak{y}_{1} D_{q} \mathfrak{F}\left(t \mathfrak{r}+(1-t) \mathfrak{y}_{1}\right)\right|^{s} d_{q} t\right)^{\frac{1}{s}} \\
& +\frac{\left(\mathfrak{y}_{2}-\mathfrak{r}\right)^{2}}{\mathfrak{y}_{2}-\mathfrak{y}_{1}}\left(\int_{0}^{1}|q t-\lambda|^{r} d_{q} t\right)^{\frac{1}{r}}\left(\int_{0}^{1}\left|\mathfrak{y}_{2} D_{q} \mathfrak{F}\left(t \mathfrak{r}+(1-t) \mathfrak{y}_{2}\right)\right|^{s} d_{q} t\right)^{\frac{1}{s}} .
\end{aligned}
$$

Now by applying convexity, we have

$$
\begin{aligned}
& \mid(1-\lambda) \mathfrak{F}(\mathfrak{r})+\frac{\lambda}{\mathfrak{y}_{2}-\mathfrak{y}_{1}}\left[\left(\mathfrak{r}-\mathfrak{y}_{1}\right) \mathfrak{F}\left(\mathfrak{y}_{1}\right)+\left(\mathfrak{y}_{2}-\mathfrak{r}\right) \mathfrak{F}\left(\mathfrak{y}_{2}\right)\right] \\
& -\frac{1}{\mathfrak{y}_{2}-\mathfrak{y}_{1}}\left[\int_{\mathfrak{y}_{1}}^{\mathfrak{r}}(t) \mathfrak{y}_{1} d_{q} t+\int_{\mathfrak{r}}^{\mathfrak{y}_{2}} \mathfrak{F}(t)^{\mathfrak{y}_{2}} d_{q} t\right] \mid \\
& \leq \frac{\left(\mathfrak{r}-\mathfrak{y}_{1}\right)^{2}}{\mathfrak{y}_{2}-\mathfrak{y}_{1}}\left(\int_{0}^{1}|q t-\lambda|^{r} d_{q} t\right)^{\frac{1}{r}}\left(\int_{0}^{1}\left[t\left|\mathfrak{y}_{1} D_{q} \mathfrak{F}(\mathfrak{r})\right|^{s}+(1-t)\left|\mathfrak{y}_{1} D_{q} \mathfrak{F}\left(\mathfrak{y}_{1}\right)\right|^{s}\right] d_{q} t\right)^{\frac{1}{s}} \\
& +\frac{\left(\mathfrak{y}_{2}-\mathfrak{r}\right)^{2}}{\mathfrak{y}_{2}-\mathfrak{y}_{1}}\left(\int_{0}^{1}|q t-\lambda|^{r} d_{q} t\right)^{\frac{1}{r}}\left(\int_{0}^{1}\left[t\left|\mathfrak{y}_{2} D_{q} \mathfrak{F}(\mathfrak{r})\right|^{s}+(1-t)\left|\mathfrak{y}_{2} D_{q} \mathfrak{F}\left(\mathfrak{y}_{2}\right)\right|^{s}\right] d_{q} t\right)^{\frac{1}{s}} \\
& =\frac{\left(\mathfrak{r}-\mathfrak{y}_{1}\right)^{2}}{\mathfrak{y}_{2}-\mathfrak{y}_{1}} \Delta_{7}(\lambda ; q, r)^{\frac{1}{r}}\left(\frac{\left|\mathfrak{y}_{1} D_{q} \mathfrak{F}(\mathfrak{r})\right|^{s}+q\left|\mathfrak{y}_{1} D_{q} \mathfrak{F}\left(\mathfrak{y}_{1}\right)\right|^{s}}{[2]_{q}}\right)^{\frac{1}{s}} \\
& +\frac{\left(\mathfrak{y}_{2}-\mathfrak{r}\right)^{2}}{\mathfrak{y}_{2}-\mathfrak{y}_{1}} \Delta_{7}(\lambda ; q, r)^{\frac{1}{r}}\left(\frac{\left|\mathfrak{y}_{2} D_{q} \mathfrak{F}(\mathfrak{r})\right|^{s}+q\left|\mathfrak{y}_{2} D_{q} \mathfrak{F}\left(\mathfrak{y}_{2}\right)\right|^{s}}{[2]_{q}}\right.
\end{aligned}
$$


Thus, the proof is completed.

Remark 5. In Theorem 9, we have:

(i) If we set $\lambda=0$, then we obtain the following quantum Ostrowski type integral inequality:

$$
\begin{aligned}
& \left|\mathfrak{F}(\mathfrak{r})-\frac{1}{\mathfrak{y}_{2}-\mathfrak{y}_{1}}\left[\int_{\mathfrak{y}_{1}}^{\mathfrak{r}} \mathfrak{F}(t) \mathfrak{y}_{1} d_{q} t+\int_{\mathfrak{r}}^{\mathfrak{y}_{2}} \mathfrak{F}(t)^{\mathfrak{y}_{2}} d_{q} t\right]\right| \\
& \leq \frac{q}{\mathfrak{y}_{2}-\mathfrak{y}_{1}}\left(\frac{1}{[r+1]_{q}}\right)^{\frac{1}{r}} \\
& \quad \times\left[\left(\mathfrak{r}-\mathfrak{y}_{1}\right)^{2}\left(\frac{\left|\mathfrak{y}_{1} D_{q} \mathfrak{F}(\mathfrak{r})\right|^{s}+q\left|\mathfrak{y}_{1} D_{q} \mathfrak{F}\left(\mathfrak{y}_{1}\right)\right|^{s}}{[2]_{q}}\right)^{\frac{1}{s}}\right. \\
& \left.+\left(\mathfrak{y}_{2}-\mathfrak{r}\right)^{2}\left(\frac{\left|\mathfrak{y}_{2} D_{q} \mathfrak{F}(\mathfrak{r})\right|^{s}+\left.\left.q\right|^{\mathfrak{y}_{2}} D_{q} \mathfrak{F}\left(\mathfrak{y}_{2}\right)\right|^{s}}{[2]_{q}}\right)^{\frac{1}{s}}\right],
\end{aligned}
$$

which is proved by Budak et al. in [2].

(ii) If we set $\lambda=1$ and $q \rightarrow 1^{-}$, then we recapture [28] [Theorem 5].

Corollary 4. In Theorem 8 , we have

(i) If we assume that $\mathfrak{y}_{1} D_{q} \mathfrak{F}(\mathfrak{r}), \mathfrak{y}_{2} D_{q} \mathfrak{F}(\mathfrak{r}) \leq M$, then we obtain the following new inequality:

$$
\begin{aligned}
& \mid(1-\lambda) \mathfrak{F}(\mathfrak{r})+\frac{\lambda}{\mathfrak{y}_{2}-\mathfrak{y}_{1}}\left[\left(\mathfrak{r}-\mathfrak{y}_{1}\right) \mathfrak{F}\left(\mathfrak{y}_{1}\right)+\left(\mathfrak{y}_{2}-\mathfrak{r}\right) \mathfrak{F}\left(\mathfrak{y}_{2}\right)\right] \\
& -\frac{1}{\mathfrak{y}_{2}-\mathfrak{y}_{1}}\left[\int_{\mathfrak{y}_{1}}^{\mathfrak{r}} \mathfrak{F}(t) \mathfrak{y}_{1} d_{q} t+\int_{\mathfrak{r}}^{\mathfrak{y}_{2}} \mathfrak{F}(t)^{\mathfrak{y}_{2}} d_{q} t\right] \mid \\
& \leq \frac{M \Delta_{7}(\lambda ; q, r)^{\frac{1}{r}}}{\mathfrak{y}_{2}-\mathfrak{y}_{1}}\left[\left(\mathfrak{r}-\mathfrak{y}_{1}\right)^{2}+\left(\mathfrak{y}_{2}-\mathfrak{r}\right)^{2}\right] .
\end{aligned}
$$

(ii) If we set $\lambda=0$ and $\mathfrak{r}=\frac{\mathfrak{y}_{1}+\mathfrak{y}_{2}}{2}$, then we obtain the following quantum midpoint type inequality:

$$
\begin{aligned}
& \mid \mathfrak{F}\left(\frac{\mathfrak{y}_{1}+\mathfrak{y}_{2}}{2}\right)-\frac{1}{\mathfrak{y}_{2}-\mathfrak{y}_{1}}\left[\int_{\mathfrak{y}_{1}}^{\frac{\mathfrak{y}_{1}+\mathfrak{y}_{2}}{2}} \mathfrak{F}(t) \mathfrak{y}_{1} d_{q} t+\int_{\frac{\mathfrak{y}_{1}+\mathfrak{y}_{2}}{2}}^{\mathfrak{y}_{2}} \mathfrak{F}(t) \mathfrak{y}_{2} d_{q} t\right] \\
& \leq \frac{q\left(\mathfrak{y}_{2}-\mathfrak{y}_{1}\right)}{4}\left(\frac{1}{[r+1]_{q}}\right)^{\frac{1}{r}} \\
& \quad \times\left[\left(\frac{\left|\mathfrak{y}_{1} D_{q} \mathfrak{F}\left(\frac{\mathfrak{y}_{1}+\mathfrak{y}_{2}}{2}\right)\right|^{s}+q\left|\mathfrak{y}_{1} D_{q} \mathfrak{F}\left(\mathfrak{y}_{1}\right)\right|^{s}}{[2]_{q}}\right)^{\frac{1}{s}}\right. \\
& \left.+\left(\frac{\left|\mathfrak{y}_{2} D_{q} \mathfrak{F}\left(\frac{\mathfrak{y}_{1}+\mathfrak{y}_{2}}{2}\right)\right|^{s}+\left.\left.q\right|^{\mathfrak{y}_{2}} D_{q} \mathfrak{F}\left(\mathfrak{y}_{2}\right)\right|^{s}}{[2]_{q}}\right)^{\frac{1}{s}}\right]
\end{aligned}
$$


(iii) If we set $\lambda=1$ and $\mathfrak{r}=\frac{\mathfrak{y}_{1}+\mathfrak{y}_{2}}{2}$, then we obtain the following quantum trapezoidal type inequality:

$$
\begin{aligned}
& \left|\frac{\mathfrak{F}\left(\mathfrak{y}_{1}\right)+\mathfrak{F}\left(\mathfrak{y}_{2}\right)}{2}-\frac{1}{\mathfrak{y}_{2}-\mathfrak{y}_{1}}\left[\int_{\mathfrak{y}_{1}}^{\frac{\mathfrak{y}_{1}+\mathfrak{y}_{2}}{2}} \mathfrak{F}(t)_{\mathfrak{y}_{1}} d_{q} t+\int_{\frac{\mathfrak{y}_{1}+\mathfrak{y}_{2}}{2}}^{\mathfrak{y}_{2}} \mathfrak{F}(t)^{\mathfrak{y}_{2}} d_{q} t\right]\right| \\
& \leq \frac{\left(\mathfrak{y}_{2}-\mathfrak{y}_{1}\right)}{4} \Delta_{7}(1 ; q, r)^{\frac{1}{r}}\left[\left(\frac{\left|\mathfrak{y}_{1} D_{q} \mathfrak{F}(\mathfrak{r})\right|^{\mathcal{S}}+q\left|\mathfrak{y}_{1} D_{q} \mathfrak{F}\left(\mathfrak{y}_{1}\right)\right|^{\mathcal{S}}}{[2]_{q}}\right)^{\frac{1}{s}}\right. \\
& \left.\quad+\left(\frac{\left|\mathfrak{y}_{2} D_{q} \mathfrak{F}(\mathfrak{r})\right|^{\mathcal{S}}+q\left|\mathfrak{y}_{2} D_{q} \mathfrak{F}\left(\mathfrak{y}_{2}\right)\right|^{S}}{[2]_{q}}\right)^{\frac{1}{S}}\right]
\end{aligned}
$$

(iv) If we set $\lambda=\frac{1}{3}$ and $\mathfrak{r}=\frac{\mathfrak{y}_{1}+\mathfrak{y}_{2}}{2}$, then we obtain the following quantum Simpson's type inequality:

$$
\begin{aligned}
& \mid \frac{1}{6}\left[\mathfrak{F}\left(\mathfrak{y}_{1}\right)+4 \mathfrak{F}\left(\frac{\mathfrak{y}_{1}+\mathfrak{y}_{2}}{2}\right)+\mathfrak{F}\left(\mathfrak{y}_{2}\right)\right] \\
& -\frac{1}{\mathfrak{y}_{2}-\mathfrak{y}_{1}}\left[\int_{\mathfrak{y}_{1}}^{\frac{\mathfrak{y}_{1}+\mathfrak{y}_{2}}{2}} \mathfrak{F}(t) \mathfrak{y}_{1} d_{q} t+\int_{\frac{\mathfrak{y}_{1}+\mathfrak{y}_{2}}{2}}^{\mathfrak{y}_{2}} \mathfrak{F}(t)^{\mathfrak{y}_{2}} d_{q} t\right] \mid \\
& \leq \frac{\left(\mathfrak{y}_{2}-\mathfrak{y}_{1}\right)}{4} \Delta_{7}\left(\frac{1}{3} ; q, r\right)^{\frac{1}{r}}\left[\left(\frac{\left|\mathfrak{y}_{1} D_{q} \mathfrak{F}(\mathfrak{r})\right|^{s}+q\left|\mathfrak{y}_{1} D_{q} \mathfrak{F}\left(\mathfrak{y}_{1}\right)\right|^{s}}{[2]_{q}}\right)^{\frac{1}{s}}\right. \\
& \left.\quad+\left(\frac{\left|\mathfrak{y}_{2} D_{q} \mathfrak{F}(\mathfrak{r})\right|^{s}+q\left|\mathfrak{y}_{2} D_{q} \mathfrak{F}\left(\mathfrak{y}_{2}\right)\right|^{s}}{[2]_{q}}\right)^{\frac{1}{s}}\right]
\end{aligned}
$$

Remark 6. We can establish more inequalities of Bullen type and Hermite-Hadamard type with different choices of the parameters and using Young's inequality and we left them for the readers.

\section{Examples}

In this section, we provide some examples to support the main theorems.

Example 1. For a convex mapping $\mathfrak{F}:[0,2] \rightarrow \mathbb{R}$ is defined by $\mathfrak{F}(\mathfrak{r})=\mathfrak{r}+4$. From Theorem 7 with $q=\frac{3}{4}, \lambda=1$ and $\mathfrak{r}=\frac{\mathfrak{y}_{1}+\mathfrak{y}_{2}}{2}$, the left side of (13) becomes

$$
\begin{aligned}
& \mid(1-\lambda) \mathfrak{F}(\mathfrak{r})+\frac{\lambda}{\mathfrak{y}_{2}-\mathfrak{y}_{1}}\left[\left(\mathfrak{r}-\mathfrak{y}_{1}\right) \mathfrak{F}\left(\mathfrak{y}_{1}\right)+\left(\mathfrak{y}_{2}-\mathfrak{r}\right) \mathfrak{F}\left(\mathfrak{y}_{2}\right)\right] \\
& -\frac{1}{\mathfrak{y}_{2}-\mathfrak{y}_{1}}\left[\int_{\mathfrak{y}_{1}}^{\mathfrak{r}} \mathfrak{F}(t) \mathfrak{y}_{1} d_{q} t+\int_{\mathfrak{r}}^{\mathfrak{y}_{2}} \mathfrak{F}(t){ }^{\mathfrak{y}_{2}} d_{q} t\right] \mid \\
= & \left|\frac{\mathfrak{F}(0)+\mathfrak{F}(2)}{2}-\frac{1}{2}\left[\int_{0}^{1} \mathfrak{F}(t){ }_{0} d_{\frac{3}{4}} t+\int_{1}^{2} \mathfrak{F}(t)^{2} d_{\frac{3}{4}} t\right]\right| \\
\approx & \left|5-\frac{1}{2}[4.5714+5.4286]\right|=0,
\end{aligned}
$$


and the right side of (13) becomes

$$
\begin{aligned}
& \frac{\left(\mathfrak{r}-\mathfrak{y}_{1}\right)^{2}}{\mathfrak{y}_{2}-\mathfrak{y}_{1}}\left(\left.\Delta_{1}(\lambda ; q)\right|_{\mathfrak{y}_{1}} D_{q} \mathfrak{F}(\mathfrak{r})\left|+\Delta_{2}(\lambda ; q)\right|_{\mathfrak{y}_{1}} D_{q} \mathfrak{F}\left(\mathfrak{y}_{1}\right) \mid\right) \\
& \quad+\frac{\left(\mathfrak{y}_{2}-\mathfrak{r}\right)^{2}}{\mathfrak{y}_{2}-\mathfrak{y}_{1}}\left(\left.\Delta_{1}(\lambda ; q)\right|^{\mathfrak{y}_{2}} D_{q} \mathfrak{F}(\mathfrak{r})\left|+\Delta_{2}(\lambda ; q)\right|^{\mathfrak{y}_{2}} D_{q} \mathfrak{F}\left(\mathfrak{y}_{2}\right) \mid\right) \\
& =\frac{1}{2}\left[\left(\Delta_{1}\left(1 ; \frac{3}{4}\right)\left|{ }_{0} D_{\frac{3}{4}} \mathfrak{F}(1)\right|+\Delta_{2}\left(1 ; \frac{3}{4}\right)\left|{ }_{0} D_{\frac{3}{4}} \mathfrak{F}(0)\right|\right)\right. \\
& \left.\quad+\left(\Delta_{1}\left(1 ; \frac{3}{4}\right)\left|{ }^{2} D_{\frac{3}{4}} \mathfrak{F}(1)\right|+\left.\Delta_{2}\left(1 ; \frac{3}{4}\right)\right|^{2} D_{\frac{3}{4}} \mathfrak{F}(2) \mid\right)\right] \\
& \approx \frac{1}{2}[(0.2471 \cdot|1|+0.3243 \cdot|1|)+(0.2471 \cdot|1|+0.3243 \cdot|1|)]=0.5714 .
\end{aligned}
$$

It is clear that

$$
0<0.5714,
$$

which demonstrates the result described in Theorem 7.

Example 2. For a convex mapping $\mathfrak{F}:[0,2] \rightarrow \mathbb{R}$ is defined by $\mathfrak{F}(\mathfrak{r})=\mathfrak{r}+4$. From Theorem 8 with $q=\frac{3}{4}, \lambda=1, s=3$ and $\mathfrak{r}=\frac{\mathfrak{y}_{1}+\mathfrak{y}_{2}}{2}$, the left side of (15) becomes

$$
\begin{aligned}
& \mid(1-\lambda) \mathfrak{F}(\mathfrak{r})+\frac{\lambda}{\mathfrak{y}_{2}-\mathfrak{y}_{1}}\left[\left(\mathfrak{r}-\mathfrak{y}_{1}\right) \mathfrak{F}\left(\mathfrak{y}_{1}\right)+\left(\mathfrak{y}_{2}-\mathfrak{r}\right) \mathfrak{F}\left(\mathfrak{y}_{2}\right)\right] \\
& -\frac{1}{\mathfrak{y}_{2}-\mathfrak{y}_{1}}\left[\int_{\mathfrak{y}_{1}}^{\mathfrak{r}} \mathfrak{F}(t) \mathfrak{y}_{1} d_{q} t+\int_{\mathfrak{r}}^{\mathfrak{y}_{2}} \mathfrak{F}(t) \mathfrak{y}_{2} d_{q} t\right] \mid \\
= & \left|\frac{\mathfrak{F}(0)+\mathfrak{F}(2)}{2}-\frac{1}{2}\left[\int_{0}^{1} \mathfrak{F}(t)_{0} d_{\frac{3}{4}} t+\int_{1}^{2} \mathfrak{F}(t)^{2} d_{\frac{3}{4}} t\right]\right| \\
\approx & \left|5-\frac{1}{2}[4.5714+5.4286]\right|=0,
\end{aligned}
$$

and the right side of (15) becomes

$$
\begin{aligned}
& \Delta_{5}(\lambda ; q)^{1-\frac{1}{s}}\left[\frac{\left(\mathfrak{r}-\mathfrak{y}_{1}\right)^{2}}{\mathfrak{y}_{2}-\mathfrak{y}_{1}}\left(\Delta_{1}(\lambda ; q)\left|\mathfrak{y}_{1} D_{q} \mathfrak{F}(\mathfrak{r})\right|^{s}+\left.\left.\Delta_{2}(\lambda ; q)\right|_{\mathfrak{y}_{1}} D_{q} \mathfrak{F}\left(\mathfrak{y}_{1}\right)\right|^{s}\right)^{\frac{1}{s}}\right. \\
& \left.+\frac{\left(\mathfrak{y}_{2}-\mathfrak{r}\right)^{2}}{\mathfrak{y}_{2}-\mathfrak{y}_{1}}\left(\left.\left.\Delta_{1}(\lambda ; q)\right|^{\mathfrak{y}_{2}} D_{q} \mathfrak{F}(\mathfrak{r})\right|^{s}+\Delta_{2}(\lambda ; q)\left|\mathfrak{y}_{2} D_{q} \mathfrak{F}\left(\mathfrak{y}_{2}\right)\right|^{s}\right)^{\frac{1}{s}}\right] \\
& =\Delta_{5}(\lambda ; q)^{1-\frac{1}{3}} \cdot \frac{1}{2}\left[\left(\Delta_{1}\left(1 ; \frac{3}{4}\right)\left|{ }_{0} D_{\frac{3}{4}} \mathfrak{F}(1)\right|^{3}+\Delta_{2}\left(1 ; \frac{3}{4}\right)\left|{ }_{0} D_{\frac{3}{4}} \mathfrak{F}(0)\right|^{3}\right)^{\frac{1}{3}}\right. \\
& \left.\quad+\left(\Delta_{1}\left(1 ; \frac{3}{4}\right)\left|{ }^{2} D_{\frac{3}{4}} \mathfrak{F}(1)\right|^{3}+\left.\left.\Delta_{2}\left(1 ; \frac{3}{4}\right)\right|^{2} D_{\frac{3}{4}} \mathfrak{F}(2)\right|^{3}\right)^{\frac{1}{3}}\right] \\
& \approx 0.5714 \cdot \frac{1}{2}\left[\left(0.2471 \cdot|1|^{3}+0.3243 \cdot|1|^{3}\right)^{\frac{1}{3}}+\left(0.2471 \cdot|1|^{3}+0.3243 \cdot|1|^{3}\right)^{\frac{1}{3}}\right] \\
& =0.4742 .
\end{aligned}
$$

It is clear that

$$
0<0.4742,
$$

which demonstrates the result described in Theorem 8. 


\section{Applications to Special Means of Real Numbers}

For any positive number $\mathfrak{y}_{1}, \mathfrak{y}_{2} \in \mathbb{R}$, we consider the following means:

(i) The Arithmetic mean:

$$
\mathcal{A}\left(\mathfrak{y}_{1}, \mathfrak{y}_{2}\right)=\frac{\mathfrak{y}_{1}+\mathfrak{y}_{2}}{2}
$$

(ii) The Harmonic mean:

$$
\mathcal{H}\left(\mathfrak{y}_{1}, \mathfrak{y}_{2}\right)=\frac{2 \mathfrak{y}_{1} \mathfrak{y}_{2}}{\mathfrak{y}_{1}+\mathfrak{y}_{2}}
$$

(iii) The Geometric mean:

$$
\mathcal{G}\left(\mathfrak{y}_{1}, \mathfrak{y}_{2}\right)=\sqrt{\mathfrak{y}_{1} \mathfrak{y}_{2}}
$$

Proposition 1. For $\mathfrak{y}_{1}, \mathfrak{y}_{2} \in \mathbb{R}$ with $\mathfrak{y}_{1}<\mathfrak{y}_{2}$, the following inequality holds:

$$
\begin{aligned}
& \left|\mathcal{A}^{k}\left(\mathfrak{y}_{1}, \mathfrak{y}_{2}\right)-\frac{2}{\mathfrak{y}_{2}-\mathfrak{y}_{1}} \mathcal{A}\left(\Theta_{1}, \Theta_{2}\right)\right| \\
& \leq M\left(\mathfrak{y}_{2}-\mathfrak{y}_{1}\right) \mathcal{A}\left(\Delta_{1}(0 ; q), \Delta_{2}(0 ; q)\right),
\end{aligned}
$$

where

$$
\begin{aligned}
& \Theta_{1}=(1-q)\left(\mathfrak{y}_{2}-\mathfrak{y}_{1}\right) \sum_{n=0}^{\infty} q^{n}\left(q^{n}\left(\mathcal{A}\left(\mathfrak{y}_{1}, \mathfrak{y}_{2}\right)\right)+\left(1-q^{n}\right) \mathfrak{y}_{1}\right)^{k} \\
& \Theta_{2}=(1-q)\left(\mathfrak{y}_{2}-\mathfrak{y}_{1}\right) \sum_{n=0}^{\infty} q^{n}\left(q^{n}\left(\mathcal{A}\left(\mathfrak{y}_{1}, \mathfrak{y}_{2}\right)\right)+\left(1-q^{n}\right) \mathfrak{y}_{2}\right)^{k}
\end{aligned}
$$

Proof. The inequality (14) for function $\mathfrak{F}(t)=t^{k}, \lambda=0$ and $\mathfrak{r}=\frac{\mathfrak{y}_{1}+\mathfrak{y}_{2}}{2}$ leads to the required result.

Proposition 2. For $\mathfrak{y}_{1}, \mathfrak{y}_{2} \in \mathbb{R}$ with $\mathfrak{y}_{1}<\mathfrak{y}_{2}$, the following inequality holds:

$$
\begin{aligned}
& \left|\mathcal{A}\left(\mathfrak{y}_{1}^{k}, \mathfrak{y}_{2}^{k}\right)-\frac{2}{\mathfrak{y}_{2}-\mathfrak{y}_{1}} \mathcal{A}\left(\Theta_{1}, \Theta_{2}\right)\right| \\
& \leq M\left(\mathfrak{y}_{2}-\mathfrak{y}_{1}\right) \mathcal{A}\left(\Delta_{1}(1 ; q), \Delta_{2}(1 ; q)\right),
\end{aligned}
$$

where

$$
\begin{aligned}
& \Theta_{1}=(1-q)\left(\mathfrak{y}_{2}-\mathfrak{y}_{1}\right) \sum_{n=0}^{\infty} q^{n}\left(q^{n}\left(\mathcal{A}\left(\mathfrak{y}_{1}, \mathfrak{y}_{2}\right)\right)+\left(1-q^{n}\right) \mathfrak{y}_{1}\right)^{k} \\
& \Theta_{2}=(1-q)\left(\mathfrak{y}_{2}-\mathfrak{y}_{1}\right) \sum_{n=0}^{\infty} q^{n}\left(q^{n}\left(\mathcal{A}\left(\mathfrak{y}_{1}, \mathfrak{y}_{2}\right)\right)+\left(1-q^{n}\right) \mathfrak{y}_{2}\right)^{k}
\end{aligned}
$$

Proof. The inequality (14) for function $\mathfrak{F}(t)=t^{k}, \lambda=1$ and $\mathfrak{r}=\frac{\mathfrak{y}_{1}+\mathfrak{y}_{2}}{2}$ leads to the required result.

Proposition 3. For $\mathfrak{y}_{1}, \mathfrak{y}_{2} \in \mathbb{R}$ with $\mathfrak{y}_{1}<\mathfrak{y}_{2}$, the following inequality holds:

$$
\begin{aligned}
& \left|\frac{\mathcal{H}\left(\mathfrak{y}_{1}, \mathfrak{y}_{2}\right)}{\mathcal{G}\left(\mathfrak{y}_{1}, \mathfrak{y}_{2}\right)}-\frac{2}{\mathfrak{y}_{2}-\mathfrak{y}_{1}} \mathcal{A}\left(\Theta_{3}, \Theta_{4}\right)\right| \\
& \leq M\left(\mathfrak{y}_{2}-\mathfrak{y}_{1}\right) \mathcal{A}\left(\Delta_{1}(0 ; q), \Delta_{2}(0 ; q)\right),
\end{aligned}
$$


where

$$
\begin{aligned}
& \Theta_{3}=(1-q)\left(\mathfrak{y}_{2}-\mathfrak{y}_{1}\right) \sum_{n=0}^{\infty} \frac{q^{n}}{\left(q^{n}\left(\mathcal{A}\left(\mathfrak{y}_{1}, \mathfrak{y}_{2}\right)\right)+\left(1-q^{n}\right) \mathfrak{y}_{1}\right)}, \\
& \Theta_{4}=(1-q)\left(\mathfrak{y}_{2}-\mathfrak{y}_{1}\right) \sum_{n=0}^{\infty} \frac{q^{n}}{\left(q^{n}\left(\mathcal{A}\left(\mathfrak{y}_{1}, \mathfrak{y}_{2}\right)\right)+\left(1-q^{n}\right) \mathfrak{y}_{2}\right)} .
\end{aligned}
$$

Proof. The inequality (14) for function $\mathfrak{F}(t)=\frac{1}{t}, \lambda=0$ and $\mathfrak{r}=\frac{\mathfrak{y}_{1}+\mathfrak{y}_{2}}{2}$ leads to the required result.

Proposition 4. For $\mathfrak{y}_{1}, \mathfrak{y}_{2} \in \mathbb{R}$ with $\mathfrak{y}_{1}<\mathfrak{y}_{2}$, the following inequality holds:

$$
\begin{aligned}
& \left|\mathcal{H}^{-1}\left(\mathfrak{y}_{1}, \mathfrak{y}_{2}\right)-\frac{2}{\mathfrak{y}_{2}-\mathfrak{y}_{1}} \mathcal{A}\left(\Theta_{3}, \Theta_{4}\right)\right| \\
& \leq M\left(\mathfrak{y}_{2}-\mathfrak{y}_{1}\right) \mathcal{A}\left(\Delta_{1}(1 ; q), \Delta_{2}(1 ; q)\right),
\end{aligned}
$$

where

$$
\begin{aligned}
& \Theta_{3}=(1-q)\left(\mathfrak{y}_{2}-\mathfrak{y}_{1}\right) \sum_{n=0}^{\infty} \frac{q^{n}}{\left(q^{n}\left(\mathcal{A}\left(\mathfrak{y}_{1}, \mathfrak{y}_{2}\right)\right)+\left(1-q^{n}\right) \mathfrak{y}_{1}\right)}, \\
& \Theta_{4}=(1-q)\left(\mathfrak{y}_{2}-\mathfrak{y}_{1}\right) \sum_{n=0}^{\infty} \frac{q^{n}}{\left(q^{n}\left(\mathcal{A}\left(\mathfrak{y}_{1}, \mathfrak{y}_{2}\right)\right)+\left(1-q^{n}\right) \mathfrak{y}_{2}\right)} .
\end{aligned}
$$

Proof. The inequality (14) for function $\mathfrak{F}(t)=\frac{1}{t}, \lambda=1$ and $\mathfrak{r}=\frac{\mathfrak{y}_{1}+\mathfrak{y}_{2}}{2}$ leads to the required result.

Proposition 5. For $\mathfrak{y}_{1}, \mathfrak{y}_{2} \in \mathbb{R}$ with $\mathfrak{y}_{1}<\mathfrak{y}_{2}$, the following inequality holds:

$$
\begin{aligned}
& \left|\mathcal{A}^{k}\left(\mathfrak{y}_{1}, \mathfrak{y}_{2}\right)-\frac{2}{\mathfrak{y}_{2}-\mathfrak{y}_{1}} \mathcal{A}\left(\Theta_{1}, \Theta_{2}\right)\right| \\
& \leq 2^{\frac{1-s}{s}} M\left(\mathfrak{y}_{2}-\mathfrak{y}_{1}\right) \Delta_{5}(0 ; q)^{1-\frac{1}{s}} \mathcal{A}^{\frac{1}{s}}\left(\Delta_{1}(0 ; q), \Delta_{2}(0 ; q)\right),
\end{aligned}
$$

where

$$
\begin{aligned}
& \Theta_{1}=(1-q)\left(\mathfrak{y}_{2}-\mathfrak{y}_{1}\right) \sum_{n=0}^{\infty} q^{n}\left(q^{n}\left(\mathcal{A}\left(\mathfrak{y}_{1}, \mathfrak{y}_{2}\right)\right)+\left(1-q^{n}\right) \mathfrak{y}_{1}\right)^{k}, \\
& \Theta_{2}=(1-q)\left(\mathfrak{y}_{2}-\mathfrak{y}_{1}\right) \sum_{n=0}^{\infty} q^{n}\left(q^{n}\left(\mathcal{A}\left(\mathfrak{y}_{1}, \mathfrak{y}_{2}\right)\right)+\left(1-q^{n}\right) \mathfrak{y}_{2}\right)^{k} .
\end{aligned}
$$

Proof. The inequality (16) for function $\mathfrak{F}(t)=t^{k}, \lambda=0$ and $\mathfrak{r}=\frac{\mathfrak{y}_{1}+\mathfrak{y}_{2}}{2}$ leads to the required result.

Proposition 6. For $\mathfrak{y}_{1}, \mathfrak{y}_{2} \in \mathbb{R}$ with $\mathfrak{y}_{1}<\mathfrak{y}_{2}$, the following inequality holds:

$$
\begin{aligned}
& \left|\mathcal{A}\left(\mathfrak{y}_{1}^{k}, \mathfrak{y}_{2}^{k}\right)-\frac{2}{\mathfrak{y}_{2}-\mathfrak{y}_{1}} \mathcal{A}\left(\Theta_{1}, \Theta_{2}\right)\right| \\
& \leq 2^{\frac{1-s}{s}} M\left(\mathfrak{y}_{2}-\mathfrak{y}_{1}\right) \Delta_{5}(1 ; q)^{1-\frac{1}{s}} \mathcal{A}^{\frac{1}{s}}\left(\Delta_{1}(1 ; q), \Delta_{2}(1 ; q)\right),
\end{aligned}
$$


where

$$
\begin{aligned}
& \Theta_{1}=(1-q)\left(\mathfrak{y}_{2}-\mathfrak{y}_{1}\right) \sum_{n=0}^{\infty} q^{n}\left(q^{n}\left(\mathcal{A}\left(\mathfrak{y}_{1}, \mathfrak{y}_{2}\right)\right)+\left(1-q^{n}\right) \mathfrak{y}_{1}\right)^{k}, \\
& \Theta_{2}=(1-q)\left(\mathfrak{y}_{2}-\mathfrak{y}_{1}\right) \sum_{n=0}^{\infty} q^{n}\left(q^{n}\left(\mathcal{A}\left(\mathfrak{y}_{1}, \mathfrak{y}_{2}\right)\right)+\left(1-q^{n}\right) \mathfrak{y}_{2}\right)^{k} .
\end{aligned}
$$

Proof. The inequality (16) for function $\mathfrak{F}(t)=t^{k}, \lambda=1$ and $\mathfrak{r}=\frac{\mathfrak{y}_{1}+\mathfrak{y}_{2}}{2}$ leads to the required result.

Proposition 7. For $\mathfrak{y}_{1}, \mathfrak{y}_{2} \in \mathbb{R}$ with $\mathfrak{y}_{1}<\mathfrak{y}_{2}$, the following inequality holds:

$$
\begin{aligned}
& \left|\frac{\mathcal{H}\left(\mathfrak{y}_{1}, \mathfrak{y}_{2}\right)}{\mathcal{G}\left(\mathfrak{y}_{1}, \mathfrak{y}_{2}\right)}-\frac{2}{\mathfrak{y}_{2}-\mathfrak{y}_{1}} \mathcal{A}\left(\Theta_{3}, \Theta_{4}\right)\right| \\
& \leq 2^{\frac{1-s}{s}} M\left(\mathfrak{y}_{2}-\mathfrak{y}_{1}\right) \Delta_{5}(0 ; q)^{1-\frac{1}{s}} \mathcal{A}^{\frac{1}{s}}\left(\Delta_{1}(0 ; q), \Delta_{2}(0 ; q)\right),
\end{aligned}
$$

where

$$
\begin{aligned}
& \Theta_{3}=(1-q)\left(\mathfrak{y}_{2}-\mathfrak{y}_{1}\right) \sum_{n=0}^{\infty} \frac{q^{n}}{\left(q^{n}\left(\mathcal{A}\left(\mathfrak{y}_{1}, \mathfrak{y}_{2}\right)\right)+\left(1-q^{n}\right) \mathfrak{y}_{1}\right)}, \\
& \Theta_{4}=(1-q)\left(\mathfrak{y}_{2}-\mathfrak{y}_{1}\right) \sum_{n=0}^{\infty} \frac{q^{n}}{\left(q^{n}\left(\mathcal{A}\left(\mathfrak{y}_{1}, \mathfrak{y}_{2}\right)\right)+\left(1-q^{n}\right) \mathfrak{y}_{2}\right)} .
\end{aligned}
$$

Proof. The inequality (16) for function $\mathfrak{F}(t)=\frac{1}{t}, \lambda=0$ and $\mathfrak{r}=\frac{\mathfrak{y}_{1}+\mathfrak{y}_{2}}{2}$ leads to the required result.

Proposition 8. For $\mathfrak{y}_{1}, \mathfrak{y}_{2} \in \mathbb{R}$ with $\mathfrak{y}_{1}<\mathfrak{y}_{2}$, the following inequality holds:

$$
\begin{aligned}
& \left|\mathcal{H}^{-1}\left(\mathfrak{y}_{1}, \mathfrak{y}_{2}\right)-\frac{2}{\mathfrak{y}_{2}-\mathfrak{y}_{1}} \mathcal{A}\left(\Theta_{3}, \Theta_{4}\right)\right| \\
& \leq 2^{\frac{1-s}{s}} M\left(\mathfrak{y}_{2}-\mathfrak{y}_{1}\right) \Delta_{5}(1 ; q)^{1-\frac{1}{s}} \mathcal{A}^{\frac{1}{s}}\left(\Delta_{1}(1 ; q), \Delta_{2}(1 ; q)\right),
\end{aligned}
$$

where

$$
\begin{aligned}
& \Theta_{3}=(1-q)\left(\mathfrak{y}_{2}-\mathfrak{y}_{1}\right) \sum_{n=0}^{\infty} \frac{q^{n}}{\left(q^{n}\left(\mathcal{A}\left(\mathfrak{y}_{1}, \mathfrak{y}_{2}\right)\right)+\left(1-q^{n}\right) \mathfrak{y}_{1}\right)}, \\
& \Theta_{4}=(1-q)\left(\mathfrak{y}_{2}-\mathfrak{y}_{1}\right) \sum_{n=0}^{\infty} \frac{q^{n}}{\left(q^{n}\left(\mathcal{A}\left(\mathfrak{y}_{1}, \mathfrak{y}_{2}\right)\right)+\left(1-q^{n}\right) \mathfrak{y}_{2}\right)} .
\end{aligned}
$$

Proof. The inequality (16) for function $\mathfrak{F}(t)=\frac{1}{t}, \lambda=1$ and $\mathfrak{r}=\frac{\mathfrak{y}_{1}+\mathfrak{y}_{2}}{2}$ leads to the required result.

Proposition 9. For $\mathfrak{y}_{1}, \mathfrak{y}_{2} \in \mathbb{R}$ with $\mathfrak{y}_{1}<\mathfrak{y}_{2}$, the following inequality holds:

$$
\begin{aligned}
& \left|\mathcal{A}^{k}\left(\mathfrak{y}_{1}, \mathfrak{y}_{2}\right)-\frac{2}{\mathfrak{y}_{2}-\mathfrak{y}_{1}} \mathcal{A}\left(\Theta_{1}, \Theta_{2}\right)\right| \\
& \leq \frac{M\left(\mathfrak{y}_{2}-\mathfrak{y}_{1}\right) \Delta_{7}(\lambda ; q, r)^{\frac{1}{r}}}{2}
\end{aligned}
$$


where

$$
\begin{aligned}
& \Theta_{1}=(1-q)\left(\mathfrak{y}_{2}-\mathfrak{y}_{1}\right) \sum_{n=0}^{\infty} q^{n}\left(q^{n}\left(\mathcal{A}\left(\mathfrak{y}_{1}, \mathfrak{y}_{2}\right)\right)+\left(1-q^{n}\right) \mathfrak{y}_{1}\right)^{k}, \\
& \Theta_{2}=(1-q)\left(\mathfrak{y}_{2}-\mathfrak{y}_{1}\right) \sum_{n=0}^{\infty} q^{n}\left(q^{n}\left(\mathcal{A}\left(\mathfrak{y}_{1}, \mathfrak{y}_{2}\right)\right)+\left(1-q^{n}\right) \mathfrak{y}_{2}\right)^{k} .
\end{aligned}
$$

Proof. The inequality (17) for function $\mathfrak{F}(t)=t^{k}, \lambda=0$ and $\mathfrak{r}=\frac{\mathfrak{y}_{1}+\mathfrak{y}_{2}}{2}$ leads to the required result.

Proposition 10. For $\mathfrak{y}_{1}, \mathfrak{y}_{2} \in \mathbb{R}$ with $\mathfrak{y}_{1}<\mathfrak{y}_{2}$, the following inequality holds:

$$
\begin{aligned}
& \left|\mathcal{A}\left(\mathfrak{y}_{1}^{k}, \mathfrak{y}_{2}^{k}\right)-\frac{2}{\mathfrak{y}_{2}-\mathfrak{y}_{1}} \mathcal{A}\left(\Theta_{1}, \Theta_{2}\right)\right| \\
& \leq \frac{M\left(\mathfrak{y}_{2}-\mathfrak{y}_{1}\right) \Delta_{7}(\lambda ; q, r)^{\frac{1}{r}}}{2}
\end{aligned}
$$

where

$$
\begin{aligned}
& \Theta_{1}=(1-q)\left(\mathfrak{y}_{2}-\mathfrak{y}_{1}\right) \sum_{n=0}^{\infty} q^{n}\left(q^{n}\left(\mathcal{A}\left(\mathfrak{y}_{1}, \mathfrak{y}_{2}\right)\right)+\left(1-q^{n}\right) \mathfrak{y}_{1}\right)^{k}, \\
& \Theta_{2}=(1-q)\left(\mathfrak{y}_{2}-\mathfrak{y}_{1}\right) \sum_{n=0}^{\infty} q^{n}\left(q^{n}\left(\mathcal{A}\left(\mathfrak{y}_{1}, \mathfrak{y}_{2}\right)\right)+\left(1-q^{n}\right) \mathfrak{y}_{2}\right)^{k}
\end{aligned}
$$

Proof. The inequality (17) for function $\mathfrak{F}(t)=t^{k}, \lambda=1$ and $\mathfrak{r}=\frac{\mathfrak{y}_{1}+\mathfrak{y}_{2}}{2}$ leads to the required result.

Proposition 11. For $\mathfrak{y}_{1}, \mathfrak{y}_{2} \in \mathbb{R}$ with $\mathfrak{y}_{1}<\mathfrak{y}_{2}$, the following inequality holds:

$$
\begin{aligned}
& \left|\frac{\mathcal{H}\left(\mathfrak{y}_{1}, \mathfrak{y}_{2}\right)}{\mathcal{G}\left(\mathfrak{y}_{1}, \mathfrak{y}_{2}\right)}-\frac{2}{\mathfrak{y}_{2}-\mathfrak{y}_{1}} \mathcal{A}\left(\Theta_{3}, \Theta_{4}\right)\right| \\
& \leq \frac{M\left(\mathfrak{y}_{2}-\mathfrak{y}_{1}\right) \Delta_{7}(\lambda ; q, r)^{\frac{1}{r}}}{2}
\end{aligned}
$$

where

$$
\begin{aligned}
& \Theta_{3}=(1-q)\left(\mathfrak{y}_{2}-\mathfrak{y}_{1}\right) \sum_{n=0}^{\infty} \frac{q^{n}}{\left(q^{n}\left(\mathcal{A}\left(\mathfrak{y}_{1}, \mathfrak{y}_{2}\right)\right)+\left(1-q^{n}\right) \mathfrak{y}_{1}\right)}, \\
& \Theta_{4}=(1-q)\left(\mathfrak{y}_{2}-\mathfrak{y}_{1}\right) \sum_{n=0}^{\infty} \frac{q^{n}}{\left(q^{n}\left(\mathcal{A}\left(\mathfrak{y}_{1}, \mathfrak{y}_{2}\right)\right)+\left(1-q^{n}\right) \mathfrak{y}_{2}\right)} .
\end{aligned}
$$

Proof. The inequality (17) for function $\mathfrak{F}(t)=\frac{1}{t}, \lambda=0$ and $\mathfrak{r}=\frac{\mathfrak{y}_{1}+\mathfrak{y}_{2}}{2}$ leads to the required result.

Proposition 12. For $\mathfrak{y}_{1}, \mathfrak{y}_{2} \in \mathbb{R}$ with $\mathfrak{y}_{1}<\mathfrak{y}_{2}$, the following inequality holds:

$$
\begin{aligned}
& \left|\mathcal{H}^{-1}\left(\mathfrak{y}_{1}, \mathfrak{y}_{2}\right)-\frac{2}{\mathfrak{y}_{2}-\mathfrak{y}_{1}} \mathcal{A}\left(\Theta_{3}, \Theta_{4}\right)\right| \\
& \leq \frac{M\left(\mathfrak{y}_{2}-\mathfrak{y}_{1}\right) \Delta_{7}(\lambda ; q, r)^{\frac{1}{r}}}{2}
\end{aligned}
$$


where

$$
\begin{aligned}
& \Theta_{3}=(1-q)\left(\mathfrak{y}_{2}-\mathfrak{y}_{1}\right) \sum_{n=0}^{\infty} \frac{q^{n}}{\left(q^{n}\left(\mathcal{A}\left(\mathfrak{y}_{1}, \mathfrak{y}_{2}\right)\right)+\left(1-q^{n}\right) \mathfrak{y}_{1}\right)}, \\
& \Theta_{4}=(1-q)\left(\mathfrak{y}_{2}-\mathfrak{y}_{1}\right) \sum_{n=0}^{\infty} \frac{q^{n}}{\left(q^{n}\left(\mathcal{A}\left(\mathfrak{y}_{1}, \mathfrak{y}_{2}\right)\right)+\left(1-q^{n}\right) \mathfrak{y}_{2}\right)} .
\end{aligned}
$$

Proof. The inequality (17) for function $\mathfrak{F}(t)=\frac{1}{t}, \lambda=1$ and $\mathfrak{r}=\frac{\mathfrak{y}_{1}+\mathfrak{y}_{2}}{2}$ leads to the required result.

\section{Conclusions}

In this work, we proved a new parameterized quantum integral identity involving left and right quantum derivatives to prove different variants of quantum integral inequalities for quantum differentiable convex functions. We also proved that the newly established inequalities could be turned into quantum Ostrowski's type inequalities for convex functions [2], classical Ostrowski's type inequalities for convex functions [27], several classical integral inequalities for convex functions [28] and several new quantum integral inequalities such as midpoint type, trapezoidal type, Ostrowski's type and Simpson's type without having to prove each one separately. Some examples are presented to illustrate the results. It is a new and interesting problem that the researcher can obtain similar inequalities for other kinds of convexity and coordinated convexity in their future work.

Author Contributions: Conceptualization, D.Z., M.A.A., W.L., H.B. and K.N.; methodology, D.Z., M.A.A., W.L., H.B. and K.N.; formal analysis, D.Z., M.A.A., W.L., H.B. and K.N.; investigation, D.Z., M.A.A., W.L., H.B. and K.N.; writing—original draft preparation, D.Z., M.A.A., W.L., H.B. and K.N.; writing—review and editing, D.Z., M.A.A., W.L., H.B. and K.N.; visualization, D.Z., M.A.A., W.L., H.B. and K.N.; supervision, D.Z.; funding acquisition, D.Z. and K.N.; All authors have read and agreed to the published version of the manuscript.

Funding: The work was supported by Key Projects of Educational Commission of Hubei Province of China (Grant No. D20192501), Open Fund of National Cryosphere Desert Data Center of China (2021kf03), and Foundation of Hubei Normal University (Grant No. 2021YJSKCSZY06, 2021056). This work was also supported by King Mongkut's University of Technology North Bangkok. Contract no. KMUTNB-63-KNOW-21.

Data Availability Statement: Not applicable.

Acknowledgments: We would like to thank the referees for their valuable comments.

Conflicts of Interest: The authors declare no conflict of interest.

\section{References}

1. Ostrowski, A. Über die Absolutabweichung einer differentienbaren Funktionen von ihren Integralmittelwert. Comment. Math. Helv. 1938, 10, 226-227. [CrossRef]

2. Budak, H.; Ali, M.A.; Alp, N.; Chu, Y.-M. Quantum Ostrowski type integral inequalities. J. Math. Inequal. 2021, in press.

3. Karabayir, I.; Tunç, M.; Yüksel, E. On some inequalities for functions whose absolute values of the second derivatives are $\alpha-, m-$, $(\alpha, m)$-logarithmically convex. Georgian Math. J. 2015, 22, 251-257. [CrossRef]

4. Kizil, Ş.; Ardiç, M.A. Inequalities for strongly convex functions via Atangana-Baleanu Integral Operators. Turk. Sci. 2021, 6, 96-109.

5. Özdemir, M.E. New Refinements of Hadamard Integral inequlaity via kFractional Integrals for $p$-Convex Function. Turk. J. Sci. 2019, 6, 1-5.

6. Rashid, S.; Butt, S.I.; Kanwal, S.; Ahmadand, H.; Wang, M.K. Quantum integral inequalities with respect to Raina's function via coordinated generalized convex functions with applications. J. Funct. Spaces 2021, 2021, 6631474. [CrossRef]

7. Alp, N.; Sarikaya, M.Z.; Kunt, M.; İşcan, İ. q-Hermite Hadamard inequalities and quantum estimates for midpoint type inequalities via convex and quasi-convex functions. J. King Saud Univ. Sci. 2018, 30, 193-203. [CrossRef]

8. Bermudo, S.; Kórus, P.; Valdés, J.N. On $q$-Hermite-Hadamard inequalities for general convex functions. Acta Math. Hung. 2020, 162, 364-374. [CrossRef]

9. Noor, M.A.; Noor, K.I.; Awan, M.U. Some quantum estimates for Hermite-Hadamard inequalities. Appl. Math. Comput. 2015, 251, 675-679. [CrossRef] 
10. Budak, H. Some trapezoid and midpoint type inequalities for newly defined quantum integrals. Proyecciones 2021, 40, 199-215. [CrossRef]

11. Liu, W.; Hefeng, Z. Some quantum estimates of Hermite-Hadamard inequalities for convex functions. J. Appl. Anal. Comput. 2016, 7,501-522.

12. Noor, M.A.; Noor, K.I.; Awan, M.U. Some quantum integral inequalities via preinvex functions. Appl. Math. Comput. 2015, 269, 242-251. [CrossRef]

13. Vivas-Cortez, M.; Kashuri, A.; Liko, R.; Hernádez, J.E. Quantum trapezium-type inequalities using generalized $\phi$-convex functions. Axioms 2020, 9, 12. [CrossRef]

14. Wang, P.P.; Zhu, T.; Du, T.S. Some inequalities using s-preinvexity via quantum calculus. J. Interdiscip. 2021, 24, 613-636. [CrossRef]

15. Brahim, K.; Taf, S.; Rihahi, L. Some result for Hadamard-type inequalities in quantum calculus. Le Mat. 2014, 69, $243-258$.

16. Jhanthanam, S.; Tariboon, J.; Ntouyas, S.K.; Nonlaopon, K. On $q$-Hermite-Hadamard inequalities for differentiable convex functions. Mathematics 2019, 7, 632. [CrossRef]

17. Neang, P.; Nanlaopon, K.; Tariboon, J.; Ntouyas, S.K.; Agarwal, P. Some trapezoid and midpoint type inequalities via fractional $(p, q)$-calculus. Adv. Differ. Equ. 2021, 2021, 333. [CrossRef]

18. Alp, N.; Sarikaya, M.Z. Hermite Hadamard's type inequalities for co-ordinated convex functions on quantum integral. Appl. Math. E-Notes 2020, 20, 341-356.

19. Budak, H.; Ali, M.A.; Tarhanaci, M. Some new quantum Hermite-Hadamard-like inequalities for coordinated convex functions. J. Optim. Theory Appl. 2020, 186, 899-910. [CrossRef]

20. Kunt, M.; Latif, M.A.; Iscan, I.; Dragomir, S.S. Quantum Hermite-Hadamard type inequality and some estimates of quantum midpoint type inequalities for double integrals. Sigma J. Eng. Nat. Sci. 2019, 37, 207-223.

21. Ali, M.A.; Budak, H.; Zhang, Z.; Yildrim, H. Some new Simpson's type inequalities for co-ordinated convex functions in quantum calculus. Math. Meth. Appl. Sci. 2021, 44, 4515-4540. [CrossRef]

22. Kalsoom, H.; Wu, J.-D.; Hussain, S.; Latif, M.A. Simpson's type inequalities for co-ordinated convex functions on quantum calculus. Symmetry 2019, 11, 768. [CrossRef]

23. Vivas-Cortez, M.; Ali, M.A.; Kashuri, A.; Sial, I.B.; Zhang, Z. Some New Newton's Type Integral Inequalities for Co-Ordinated Convex Functions in Quantum Calculus. Symmetry 2020, 12, 1476. [CrossRef]

24. Kunt, M.; Kashuri, A.; Du, T.; Baidar, A.W. Quantum Montgomery identity and quantum estimates of Ostrowski type inequalities AIMS Math. 2020, 5, 5439-5457. [CrossRef]

25. Vivas-Cortez, M.; Kashuri, A.; Liko, R.; Hernádez, J.E. Quantum estimates of Ostrowski inequalities for generalized $\phi$-convex functions. Symmetry 2019, 11, 1513. [CrossRef]

26. Alomari, M.W. q-Bernoulli inequality. Turk. J. Sci. 2018, 3, 32-39.

27. Cerone, P.; Dragomir, S.S. Ostrowski type inequalities for functions whose derivatives satisfy certain convexity assumptions. Demonstr. Math. 2004, 37, 299-308. [CrossRef]

28. Kavurmaci, H.; Avci, M.; Özdemir, M.E. New Inequalities of Hermite-Hadamard type for convex functions with applications J. Inequal. Appl. 2011, 2011, 86. [CrossRef]

29. Tariboon, J.; Ntouyas, S.K. Quantum calculus on finite intervals and applications to impulsive difference equations. Adv. Differ. Equ. 2013, 2013, 1-19. [CrossRef]

30. Jackson, F.H. On a q-definite integrals. Q. J. Pure Appl. Math. 1910, 41, 193-203.

31. Kac, V.; Cheung, P. Quantum Calculus; Springer: Berlin/Heidelberg, Germany, 2001.

32. Ali, M.A.; Budak, H.; Nanlaopon, K.; Abdullah, Z. Simpson's and Newton's Inequalities for $(\alpha, m)$-Convex Functions via Quantum Calculus. 2021, Submitted.

33. Sial, I.B.; Mei, S.; Ali, M.A.; Nanlaopon, K. On some generalized Simpson's and Newton's inequalities for $(\alpha, m)$-convex functions in $q$-calculus. Mathematics 2021, 2021, 3266. [CrossRef] 